\title{
Characterization of molecular diversity and genome-wide mapping of loci associated with resistance to stripe rust and stem rust in Ethiopian bread wheat accessions
}

Kebede T. Muleta', Matthew N. Rouse², Sheri Rynearson'1, Xianming Chen ${ }^{3}$, Bedada G. Buta ${ }^{4}$ and Michael O. Pumphrey ${ }^{1 *}$

\begin{abstract}
Background: The narrow genetic basis of resistance in modern wheat cultivars and the strong selection response of pathogen populations have been responsible for periodic and devastating epidemics of the wheat rust diseases. Characterizing new sources of resistance and incorporating multiple genes into elite cultivars is the most widely accepted current mechanism to achieve durable varietal performance against changes in pathogen virulence. Here, we report a high-density molecular characterization and genome-wide association study (GWAS) of stripe rust and stem rust resistance in 190 Ethiopian bread wheat lines based on phenotypic data from multi-environment field trials and seedling resistance screening experiments. A total of 24,281 single nucleotide polymorphism (SNP) markers filtered from the wheat $90 \mathrm{~K}$ iSelect genotyping assay was used to survey Ethiopian germplasm for population structure, genetic diversity and marker-trait associations.
\end{abstract}

Results: Upon screening for field resistance to stripe rust in the Pacific Northwest of the United States and Ethiopia over multiple growing seasons, and against multiple races of stripe rust and stem rust at seedling stage, eight accessions displayed resistance to all tested races of stem rust and field resistance to stripe rust in all environments. Our GWAS results show 15 loci were significantly associated with seedling and adult plant resistance to stripe rust at false discovery rate (FDR)-adjusted probability $(P)<0.10$. GWAS also detected 9 additional genomic regions significantly associated (FDR-adjusted $P<0.10$ ) with seedling resistance to stem rust in the Ethiopian wheat accessions. Many of the identified resistance loci were mapped close to previously identified rust resistance genes; however, three loci on the short arms of chromosomes $5 \mathrm{~A}$ and $7 \mathrm{~B}$ for stripe rust resistance and two on chromosomes 3B and 7B for stem rust resistance may be novel.

Conclusion: Our results demonstrate that considerable genetic variation resides within the landrace accessions that can be utilized to broaden the genetic base of rust resistance in wheat breeding germplasm. The molecular markers identified in this study should be useful in efficiently targeting the associated resistance loci in marker-assisted breeding for rust resistance in Ethiopia and other countries.

Keywords: Bread wheat, Stripe rust, Stem rust, Genetic resistance, Genetic diversity, Association mapping

\footnotetext{
* Correspondence: m.pumphrey@wsu.edu

1 Department of Crop and Soil Sciences, Washington State University,

Pullman, WA 99164-6420, USA

Full list of author information is available at the end of the article
} 


\section{Background}

Stripe rust [Puccinia striiformis Westend. f. sp. tritici Erikss. (Pst)] and stem rust [Puccinia graminis Pers.:Pers. f. sp. tritici Erikss. \& E. Henn. (Pgt)] are two of the most damaging diseases of wheat worldwide [1-3]. The ability of wheat rust pathogen populations to quickly evolve new pathotypes that overcome deployed resistance genes and produce multiple cycles of urediniospores in a single season, along with their capacity for long distance dispersal, create potential for destructive epidemics in susceptible varieties under favorable conditions. At various times in history, epidemics of both diseases have caused massive losses to wheat production globally. Stripe rust has been a disease of wheat mainly in areas with cooler climates [1]. More recently, however, the emergence of aggressive races of Pst tolerant to higher temperatures has resulted in yield loss in areas normally considered too warm for serious epidemic development. These new strains of Pst are currently widespread and threatening wheat production on a global scale $[1,4,5]$. In recent years, wheat growing regions in East Africa, Central and West Asia, and the Caucasus countries experienced one of the largest stripe rust epidemics in the recent history [6]. In Ethiopia alone, this epidemic affected more than 600,000 ha of wheat and led to an expenditure of more than \$US 3.2 million on fungicides, while significant widespread losses were still realized [7].

During the last 40 years, introgression of different combinations of stem rust resistance genes and other epidemic mitigation strategies have reduced global stem rust epidemics [8, 9]. However, the discovery of race TTKSK (isolate Ug99) of Pgt in Uganda in 1998 has raised a particular concern to global wheat production and food security due to its wide virulence spectrum [10]. Currently, at least eight variants of TTKSK (the Ug99 lineage races) have been described with virulence for additional resistance genes, including Sr24, Sr36, Sr9h and SrTmp [11-14]. These races are currently spreading across eastern, southern and northern Africa as well as the Middle East [8, 14, 15]. In 2013-2014, a new stem rust race designated as TKTTF caused a severe epidemic on the variety 'Digalu' carrying SrTmp in Ethiopia [16]. The continued emergence of new virulent races of stem rust emphasizes the dynamic challenges of breeding for stem rust resistance.

To mitigate losses due to rust diseases in wheat, world wheat production has largely depended on the use of resistant wheat varieties $[1,17,18]$. The emergence of pathogen populations virulent against deployed resistance genes has caused not only tremendous yield and quality losses, but has also led to frequent replacement of the otherwise agronomically superior cultivars, as well as interference with progress in improving other important traits [17]. Achieving durable varietal performance has therefore been the primary focus of many breeding programs in wheat. Two categories of resistance genes have been widely recognized in wheat breeding for rust resistance; all-stage resistance (also called seedling resistance) and adult-plant resistance (APR) [1]. Developing cultivars carrying effective seedling resistance in combination with APR genes is more desirable to minimize the damage caused by new mutants of the pathogen $[3,17]$. The deployment of cultivars with durable rust resistance in wheat is particularly desirable in regions such as Ethiopia and the neighboring countries in East Africa that are characterized by the presence of year-round inoculum due to nearly constant wheat cropping seasons and a suitable environment. These conditions provide the pathogen not only with a continuous substrate both in area and in time due to a green bridge between the seasons, but may also result in rapid selection for virulent races of the pathogen.

Limited variation in elite germplasm may constrain deployment of diverse resistance genes in released cultivars and the capacity for countering new virulence in pathogen populations [19, 20]. Landraces and other germplasm collections maintained in germplasm banks may provide access to diverse alleles for disease resistance and reverse the trend of genetic diversity erosion in established, elite cultivars [21, 22]. Such genetic resources are potentially untapped sources of useful genetic diversity owing to their limited use in modern plant breeding programs. The usefulness of wheat landraces as a good source of resistance to diseases in wheat have been demonstrated [23-27]. The objectives of this study were: 1) to evaluate Ethiopian bread wheat landraces and cultivars for their field and seedling resistance to stripe rust and stem rust across a range of environments and multiple races of the pathogens, 2) to assess the genetic diversity of Ethiopian bread wheat landraces and cultivars based on high-density genotyping, and 3) to conduct a genome-wide search for molecular markers associated with loci underpinning seedling and field resistance to stripe rust and stem rust diseases of wheat.

\section{Methods \\ Plant materials}

One hundred and ninety bread wheat accessions were used in this study. The panel was comprised of 124 landraces and 66 commercial cultivars and advanced breeding lines from the Ethiopian Institute of Agricultural Research (EIAR). Most of the commercial cultivars and advanced breeding lines were originally developed by the International Maize and Wheat Improvement Center (CIMMYT), some by the International Center for Agricultural Research in the Dry Areas (ICARDA), but evaluated and released as cultivars by the Ethiopian 
wheat improvement program. One hundred and ten accessions were landraces of hexaploid wheat originally from the Ethiopian Institute of Biodiversity, characterized and maintained by EIAR at Debre-Zeit Agricultural Research Center (DZARC), from where they were obtained. Fourteen landraces were obtained from the United States Department of Agriculture, Agricultural Research Service (USDA-ARS) National Small Grains Collection (NSGC) in Aberdeen, ID, USA. Two susceptible cultivars, Avocet $\mathrm{S}$ and Morocco, used in this study as checks were obtained from the USDA-ARS, Wheat Health, Genetics, and Quality Research Unit, Pullman, WA; and DZARC, respectively.

\section{Stripe rust response evaluation under field conditions}

The panel was tested for response to stripe rust infection under field conditions in four nurseries in the Pacific Northwest (PNW) of the US and in Ethiopia for three consecutive growing seasons (2012-2014). The locations in the PNW were Mount Vernon $\left(48^{\circ} 25^{\prime} 12^{\prime \prime} \mathrm{N} ; 122^{\circ} 19^{\prime}\right.$ $\left.34^{\prime \prime} \mathrm{W}\right)$, a high rainfall area located west of the Cascade

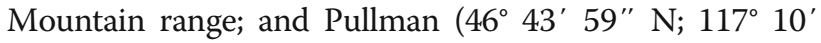
$\left.00^{\prime \prime} \mathrm{W}\right)$, a semi-arid wheat belt area located east of the Cascade Mountain range. Locations in Ethiopia included Meraro $\left(07^{\circ} 24^{\prime} 27^{\prime \prime} \mathrm{N} ; 39^{\circ} 14^{\prime} 56^{\prime \prime} \mathrm{E}\right)$ and Arsi Robe $\left(07^{\circ}\right.$ $53^{\prime} 02^{\prime \prime} \mathrm{N} ; 39^{\circ} 37^{\prime} 40^{\prime \prime}$ E), sub-stations of Kulumsa Agricultural Research Center of the Ethiopian Institute of Agricultural Research, representing relatively high annual rainfall and cool temperatures. Each nursery location is subject to high disease pressure on an annual basis, but has variable Pst races and environmental conditions. The accessions were planted as single rows of non-replicated trials with repeating checks. The susceptible cultivars Avocet S (at Mount Vernon and Pullman) and Morocco (at Meraro and Arsi Robe) were planted every 20 rows and on each side of the plot to ensure uniform disease pressure across the experimental plots. Accessions were evaluated for response Pst under natural disease epidemics. Host response to infection [i.e. infection types (IT)] to stripe rust was estimated using a $0-9$ scale as described by Line and Qayoum [28]. Stripe rust disease severity (DS) was recorded as percent leaf area showing disease symptoms. No permits were required to carry out field experiments. In addition, the field studies did not involve endangered or protected species.

\section{Seedling resistance screening to Pst}

Seedlings of the 190 accessions were evaluated for response to five isolates representing five races of Pst under greenhouse conditions at Washington State University, Pullman, WA. Three of the races (PSTv-14, PSTv-37 and PSTv-40) were of US origin [29], one race from Ethiopia, but also detected in the USA (PSTV-41) and one race predominant only in Ethiopia (PSTv-106) were used in the study [30].
The virulence/avirulence formulae of the isolates are presented in Additional file 1. All Pst races used in this study were obtained from the USDA-ARS, Wheat Health, Genetics, and Quality Research Unit, Pullman, WA.

For the seedling test, three to five seeds of each accession were planted into pots filled with the Sunshine ${ }^{\circ}$ mix growing medium (Sun Gro Horticulture, Agawam, MA, and USA). The susceptible check, 'Avocet $S$ ' and the stripe rust single-gene differential lines were also included in each experiment. Ten-day old seedlings were inoculated with urediniospores of each race. The urediniospores were mixed with talcum to ensure uniform coverage of the spores over the leaves of the seedlings. Inoculated seedlings were kept in a dark dew chamber for $24 \mathrm{~h}$ at $10{ }^{\circ} \mathrm{C}$, and then transferred into a growth chamber with temperature programmed to change gradually between $4{ }^{\circ} \mathrm{C}$ at 2:00 am during the $8 \mathrm{~h}$ dark period and $20{ }^{\circ} \mathrm{C}$ at 2:00 pm during the 16-h light period [29]. Stripe rust infection types (IT) based on the 0-9 scale were recorded at 18-20 days after inoculation as described by Line and Qayoum [28].

\section{Seedling resistance screening to Pgt}

The Ethiopian bread wheat cultivars and landraces were evaluated for reaction to four Pgt races at the USDA-ARS Cereal Disease Laboratory (CDL), St. Paul, MN. The four stem rust races were TTKSK (isolate 04KEN156/04), TRTTF (06YEM34-1), TTTTF (01MN84A-1-2) and TKTTF (13ETH18-1) that were selected based on their differential virulence patterns and potential impact on wheat production. TRTTF is broadly virulent to stem rust resistance genes including $\operatorname{Sr} 1 R S^{\text {Amigo }}$ and was detected in Yemen and Ethiopia [31]. Race TTKSK (Ug99) has a wide virulence spectrum and is rapidly evolving in East Africa. Race TTTTF is the most virulent race from the United States, producing high infection types (ITs) on the majority of stem rust differential lines [32]. TKTTF is a new race that was responsible for the localized severe stem rust epidemic in Ethiopia in 2013-2014 due to its virulence to the stem rust resistance gene SrTmp in cultivar 'Digalu' [16]. Seedling resistance evaluations were performed as described previously by Rouse et al., 2011 [33]. Infection types (ITs) were recorded on a 0 to 4 scale according to Stakman et al., 1962 [34]. Stakman ITs were converted to a linear scale using a conversion algorithm [35]. All the Pgt races used in this study were obtained from the USDA-ARS Cereal Disease Laboratory (CDL), St. Paul, MN.

\section{Genotyping}

The 190 Ethiopian bread wheat accessions were genotyped using the 90,000 SNP Illumina Infinium assay at USDA-ARS Fargo, ND, USA. Illumina genotypic data were analyzed using Illumina GenomeStudio v2011.1 
software to optimize the SNP call rates. After removing SNPs with low-quality clustering and those with minor allele frequency (MAF) less than 5\%, 24,281 high quality SNP markers with genetic map information were used for GWAS analyses. The genetic positions of the SNP markers were based on the wheat $90 \mathrm{~K} \mathrm{SNP}$ consensus map [36]. The dataset was also filtered using a 10\% cutoff for missing data in the accessions. Accordingly, a total of 179 accessions were retained in the GWAS and population structure analyses.

For internal controls, the accessions were screened with molecular markers for known stripe rust and stem rust resistance genes, including $L r 34 / Y r 18 / P m 38$ (KASP marker wMAS000003) [37], Lr37/Yr17/Sr38 (CAPS marker Ventriup-LN2) [37], Lr67/Yr46 (Kasp856) [38], Sr31/Yr9/Lr26 (KASP 1RS:1BL_6110) [39], Sr24 (barc71) [40], Sr36 (KASP wMAS000015), [37], Sr2/Yr30 (KASP wMAS000005) [37]. Markers with a MAF greater than $5 \%$ were included in the GWAS and linkage disequilibrium analyses.

Analyses of molecular diversity and population structure Genetic diversity was analyzed based on Nei's gene diversity, which is an estimate of the probability that two randomly chosen alleles from the population are different, and polymorphism information content (PIC), which reflects the probability of polymorphism between two random samples in the germplasm, using POWERMARKER v3.25 [41, 42]. The two indices of genetic diversity were used to compare the extent of molecular diversity among the landrace collection and the contemporary Ethiopian bread wheat cultivars. A $t$-test was performed using the software package JMP Genomics v6.0 (SAS Institute, Cary, $\mathrm{NC)}$ to compare the extent of molecular diversity between landraces and elite Ethiopian cultivars.

Cluster analysis based on the neighbor joining (NJ) tree algorithm according to shared-allele distance was also used to determine the genetic structure of the accessions using the phylogenetic tree analysis package in POWERMARKER v3.25. The assessment of the branching pattern in the NJ tree was based on bootstrapping over loci with 1000 replications. FigTree program v1.4 (http:// tree.bio.ed.ac.uk/software/figtree/) was used to display the consensus bootstrap value generated by PHYLIP v3.66 [43].

Population structure was analyzed using a set of 355 random SNP markers distributed across the 21 wheat chromosomes (12-23 markers per chromosome) spaced at $>10 \mathrm{cM}$ apart. The admixture model with correlated allele frequency implemented in STRUCTURE software version 2.2.3 was applied to detect population structure in the germplasm panel [44]. The parameters were set to a burn-in of 20,000 iterations and 50,000 Monte Carlo Markov Chain (MCMC) replicates to determine K values in the range of 1 to 10 . For each $\mathrm{K}$, five independent runs were carried out. The Evanno method [45] was used to determine the likely number of subpopulations. Principal components $(\mathrm{PC})$ were inferred using the software package JMP Genomics v6.0 (SAS Institute, Cary, NC) to further analyze population sub-structuring and compare the results with results from analysis with STRUCTURE software.

\section{Linkage disequilibrium analysis}

Pairwise measures of linkage disequilibrium (LD) between SNP markers were estimated using the software package JMP Genomics v6.0 (SAS Institute, Cary, NC). LD was estimated as squared allele frequency correlation $\left(r^{2}\right)$ between pairs of intra-chromosomal SNPs with known chromosomal position. To determine the average pattern of genome-wide LD decay over genetic distance, a scatterplot of $r^{2}$ values against the corresponding genetic distance between markers was constructed. The seconddegree locally weighted polynomial regression (LOESS)based curve was fitted to estimate the extent of LD decay [46]. The critical $r^{2}$ value that indicates the demarcation beyond which LD is due to true physical linkage was determined by taking the 95th percentile of the square root of transformed $r^{2}$ data of unlinked markers [47]. The genetic distance at which the LD decay curve intersects with the critical $r^{2}$ value was used as a threshold to determine the confidence interval of significant QTL.

\section{Analyses of variance and heritability}

Variance components were estimated for stripe rust IT and DS from the field experiments with a mixed linear model using the Restricted Maximum Likelihood (REML) method [48]. Genotype, location, genotype $\mathrm{x}$ location interaction and replication (season) were considered to have random effects whereas the overall mean was considered to have fixed effect. Variance components were estimated for each location and across all locations according to the following model:

$$
y_{i j k}=\mu+g_{i}+\mathrm{k}_{j}+e_{j}+g e_{i j}+e_{i j}
$$

Where $y_{i j}$ is the observation for genotype $i$ at environment $j$ in season $\mathrm{k}, \mu$ is the overall mean, $g_{i}$ the effect of the accession $i, e_{j}$ the effect of environment $j, \mathrm{k}_{j}$ the effect of season $k, g_{e i j}$ the interaction between accession $i$ within environment $j$, and $e_{i j}$ the residual.

Heritability $\left(H^{2}\right)$ estimates were calculated for each location and across all locations as:

$$
H^{2}=\sigma_{G}^{2} /\left(\sigma_{G}^{2}+\left(\sigma_{E}^{2} / y\right)+\left(\sigma_{G X E}^{2} / y\right)+\sigma_{\text {error }}^{2} / y\right)
$$

Where $\sigma_{G}^{2}$ is the genotypic variance, $\sigma_{E}^{2}$ is the environment variance, $\sigma_{G X E}^{2}$ is the genotype by environment interaction variance, and $\sigma_{\text {error }}^{2}$ is the residual error variance and $y$ is the number years within each location or 
location by year for the estimates of heritability across all environments. Genotype adjusted means were computed based on best linear unbiased predictions (BLUPs). Pearson correlation coefficient between locations and seasons were calculated to determine the consistency of IT and DS across the environments.

\section{Marker-trait association (MTA)}

To identify loci associated with response to Pst and Pgt, genome-wide association analyses were conducted using a total of 24,281 high quality SNP markers and phenotypic traits values (IT and DS) from the field and greenhouse experiments. Marker-trait associations (MTAs) were identified using the compressed mixed linear model (CMLM) $[49,50]$ implemented in GAPIT (Genomic Association and Prediction Integrated Tool) R package [51]. The first three principal components (PC3) and a compressed relationship matrix $(\mathrm{K})$ were included as fixed and random effect, respectively. Three additional association test models were compared to the PC3 + K CMLM model for correcting population structure and kinship based on the deviances of observed probability from expected distribution in Q-Q plot were used to compare the models. The following three additional models were tested: a fixed general linear model with no correction for population structure (GLM), general linear model with the first three principal components included as a fixed covariate (PC3 GLM) and mixed linear model with compressed relationship matrix included as a random covariate (K CMLM). Association analyses were conducted for IT and DS values from each location separately and estimates of BLUPs for each location and across all environments.

\section{Results}

Phenotypic variability and estimates of heritability

Analysis of variance revealed a highly significant $(P<0.0001)$ difference among the genotypes both for individual locations and data combined across locations (Table 1). Genotype by environment interactions were significant $(P<0.01)$ for disease severity at Pullman, Mount Vernon and the combined analysis across all locations. The variance components for locations were not significant across all analyses. Based on the IT data, 21 accessions (11\%) were highly resistant to all races of Pst as seedlings and across all locations and seasons at adult plant stages. Eleven of the 21 resistant accessions were landraces, while the remaining 10 accessions were cultivars. Twenty-three accessions (13\%) showed highly susceptible reactions to all races as seedlings and across all locations and seasons at adult plant stage (Fig. 1). Heritability $\left(H^{2}\right)$ values for stripe rust IT and DS ranged from $73 \%$ to $93 \%$ (Table 1). Correlation coefficients between locations and seasons for stripe rust IT and DS are summarized in Additional file 2. The Pearson correlation coefficients for stripe rust IT and DS between the multiple locations over multiple growing seasons averaged 0.64 and 0.72 , respectively. Average correlations between seasons within locations were $0.79,0.76$ and 0.64 for IT, and 0.80 , 0.860 .68 for DS at Pullman, Mount Vernon and Ethiopia, respectively. Correlation between Pullman and Mount Vernon (over multiple seasons) (averaged 0.72 and 0.78 for IT and DS, respectively), were significantly higher than the correlation between Ethiopia and the two locations in PNW; the respective average correlation coefficients for IT and DS were 0.60 and 0.58 between Pullman and Ethiopia, and 0.54 and 0.55 between Mount Vernon and Ethiopia.

Table 1 Mean response to Puccinia striiformis f. sp. tritici infection, estimates of variance components and heritability

\begin{tabular}{|c|c|c|c|c|c|c|c|}
\hline \multirow[t]{2}{*}{ Parameter } & \multicolumn{2}{|l|}{ Pullman } & \multicolumn{2}{|c|}{ Mount Vernon } & \multirow{2}{*}{$\begin{array}{l}\text { Ethiopia } \\
\text { IT (0-9) }\end{array}$} & \multicolumn{2}{|c|}{ Across locations } \\
\hline & IT (0-9) & Severity (\%) & IT (0-9) & Severity (\%) & & IT (0-9) & Severity (\%) \\
\hline Minimum & 0.0 & 0.0 & 0.0 & 0.0 & 0.0 & 0.0 & 0.0 \\
\hline Mean & 4.2 & 41.0 & 4.2 & 43.0 & 4.8 & 4.4 & 42.0 \\
\hline Maximum & 9.0 & 100.0 & 9.0 & 100.0 & 9.0 & 0.0 & 100.0 \\
\hline$\sigma_{\mathrm{G}}^{2}$ & $2.7^{* * *}$ & $360.0^{* * * *}$ & $4.5^{* * *}$ & $612.0^{* * * *}$ & $4.1^{* * *}$ & $2.9^{* * *}$ & $451.2^{* * * *}$ \\
\hline$\sigma_{\mathrm{E}}^{2}$ & $0.9^{\text {ns }}$ & $141.0^{\mathrm{ns}}$ & $0.1^{\mathrm{ns}}$ & $4.4^{\mathrm{ns}}$ & $0.1^{\mathrm{ns}}$ & $0.3^{\text {ns }}$ & 48.1 \\
\hline$\sigma_{G X E}^{2}$ & $0.1^{\mathrm{ns}}$ & $117.0^{* * *}$ & 0.0 & $90.9^{* *}$ & $0.3^{*}$ & $0.2^{*}$ & $136.9^{* * *}$ \\
\hline$\sigma_{\text {error }}^{2}$ & 1.0 & 1.0 & $0.7^{* * *}$ & 1.0 & 1.0 & 1.8 & 1.0 \\
\hline Heritability & 0.73 & 0.74 & 0.92 & 0.93 & 0.85 & 0.89 & 0.91 \\
\hline
\end{tabular}

$\sigma_{G}^{2}=$ estimate of genotypic variance

$\sigma_{E}^{2}=$ estimate of environmental variance

$\sigma_{G E}^{2}=$ estimate of genotype $\mathrm{x}$ environment variance

$\sigma_{e}^{2}=$ estimate of residual variance; $H^{2}=$ heritability

$I T$ infection type; DS disease severity; $n$ s not significant

$* P<0.05$

${ }^{* *} P<0.01$

***P $<0.001$

**** $P<0.0001$ 

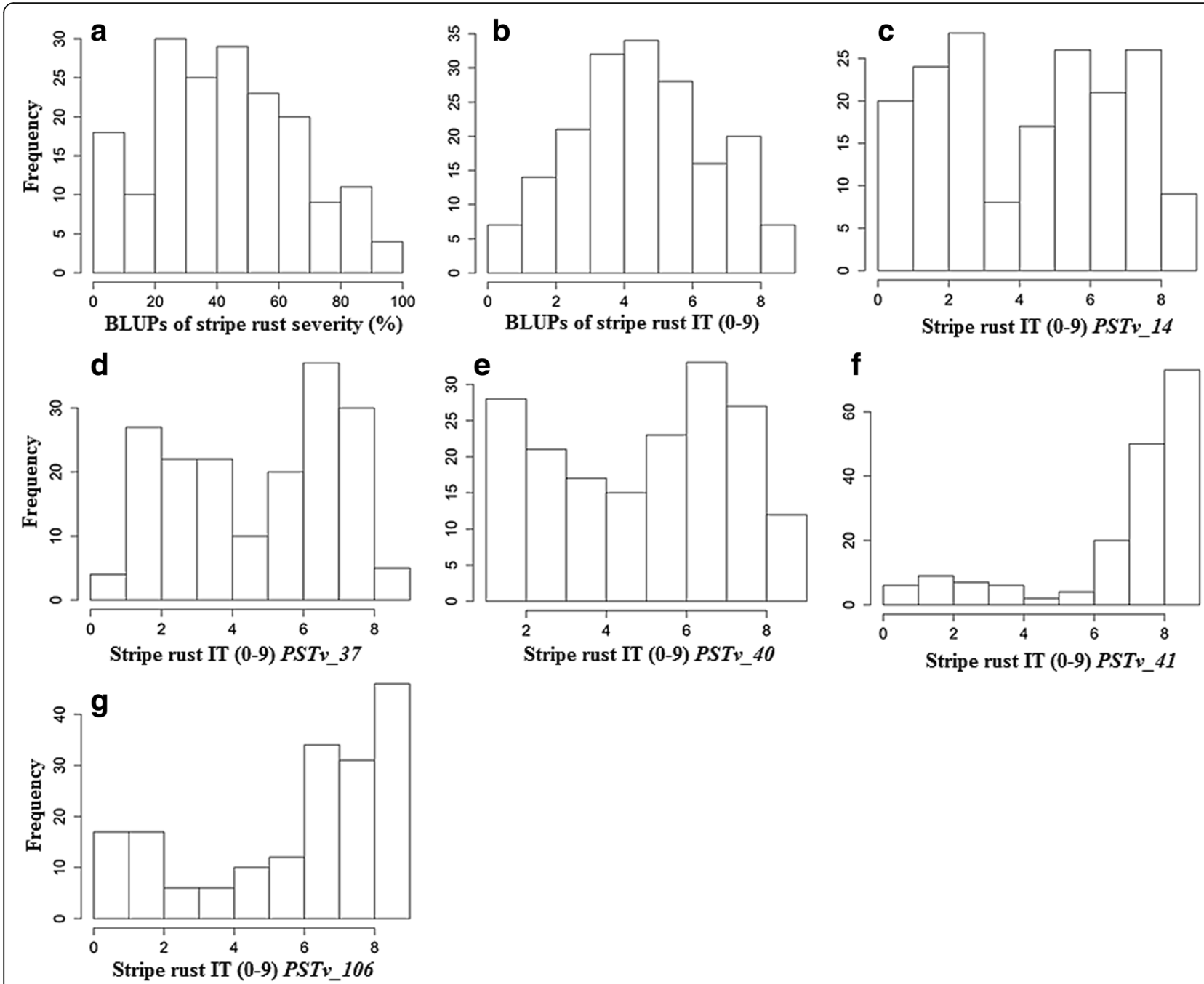

Fig. 1 Frequency distributions of stripe rust responses produced by the Ethiopian wheat accessions. $\mathbf{a}$ and $\mathbf{b}$ stripe rust infection types (IT) and disease severity (DS) under field conditions, $\mathbf{c}-\mathbf{g}$ ITs produced by seedlings when tested against the five races of Pst under greenhouse conditions

The frequency distribution of the linearized ITs produced by the four Pgt races on the Ethiopian cultivars and landraces is shown in Fig. 2. Among all accessions, 75 (41\%), 84 (46\%), 61 (34\%) and 69 (38\%) showed seedling resistance to races TTKSK, TRTTF, TTTTF and TKTTF, respectively. Fifteen (8\%) of the accessions were resistant (IT $=0$ to 23) to all four races, of which the majority were landraces. Eight accessions (5\%) shared resistance to the four races of Pgt and field resistance to Pst at all environments. Five of the 8 accessions resistant to the four races of Pgt and with field resistance to Pst at all environments were cultivars, while the remaining 3 were landraces. Additional file 3 summarizes the responses of the accessions to Pst and Pgt infection in the seedling and field resistance screenings.

\section{Molecular diversity and population structure}

Genome-specific analyses of Nei's gene diversity and PIC values were used to compare the extent of genetic variation in modern Ethiopian cultivars and accessions maintained in the germplasm collection. The analyses revealed a highly significant difference $(P<0.001)$ between cultivars and landraces in terms of both Nei's gene diversity index and PIC values. Both diversity indices were significantly higher in the landraces in 13 of the 21 wheat chromosomes (including 1A, 2D, 3A, 3B, 3D, 4A, 5A, 5B, 6A, 6D, 7A, 7B and 7D). The cultivars showed higher values of gene diversity and PIC values over the landraces only in chromosome $1 \mathrm{~B}$ and 2A. Genome-wide average Nei's gene diversity index values were 0.36 and 0.32 for the landraces and cultivars, respectively, while the PIC values were 0.29 and 0.25 for the landraces and cultivars, respectively (Fig. 3).

A neighbor-joining (NJ) phylogeny analysis based on shared allele distance showed that the Ethiopian improved varieties exhibited a high degree of genetic relatedness and were clearly distinct from the majority of landraces (Fig. 4). Based on pairwise kinship analysis 


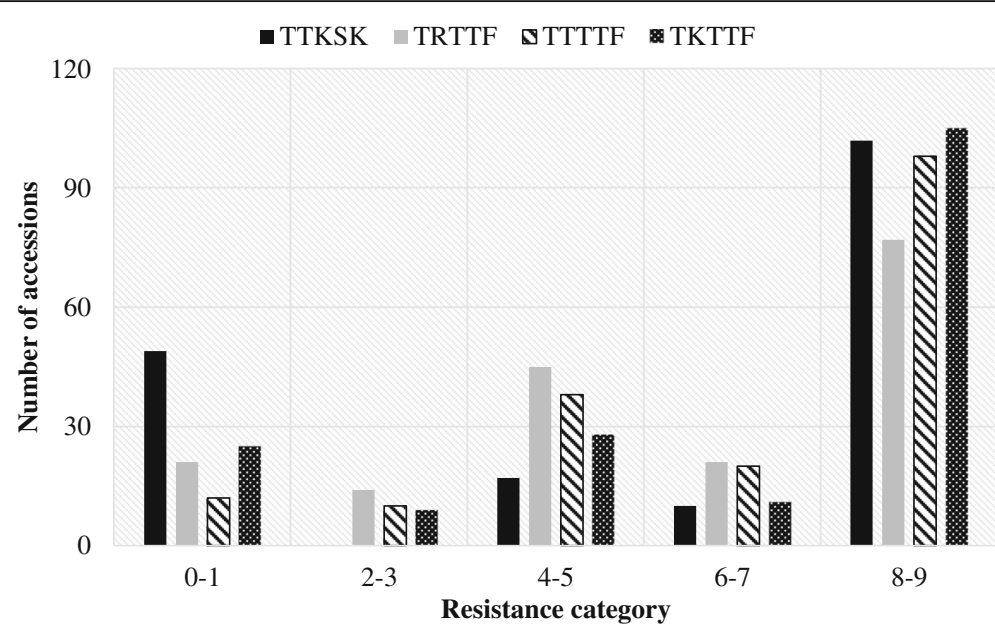

Fig. 2 Frequency distributions of stem rust infection types produced by seedlings of the Ethiopian wheat accessions

using the Fast Ward clustering algorithms, about 31\% of the released commercial cultivars showed coefficient of kinship greater than 0.9 (1.0 is exact resemblance) with each other.

Bayesian analysis of population structure yielded a strong signal for the existence of two distinct genetic clusters in the germplasm panel. With $\mathrm{K}=2$, more than $91 \%$ of the accessions were assigned to a specific cluster with membership probability higher than $75 \%$, with the remaining accessions (9\%) being classified as admixed. Kinship and principal component analysis (PCA) tended to group the accessions into three subgroups, in which all of the improved varieties were categorized into a distinct third cluster (Fig. 5). The structure analysis yielded similar clustering patterns as PCA when the second subpopulation was further analyzed separately. PCA revealed that the first and second principal component PCs accounted for $11.5 \%$ and $6.6 \%$ of the variance, respectively.

\section{Linkage disequilibrium estimation}

The extent of LD and average rate of LD decay were estimated by squared correlation coefficient $\left(r^{2}\right)$ for all pairs of SNPs along each chromosome. LD due to physical linkage, estimated as the 95th percentile of the distribution of LD $r^{2}$ between unlinked SNPs, was 0.18 . On a genome-wide level, $33.3 \%$ of all pairwise SNP loci showed LD greater than the critical value

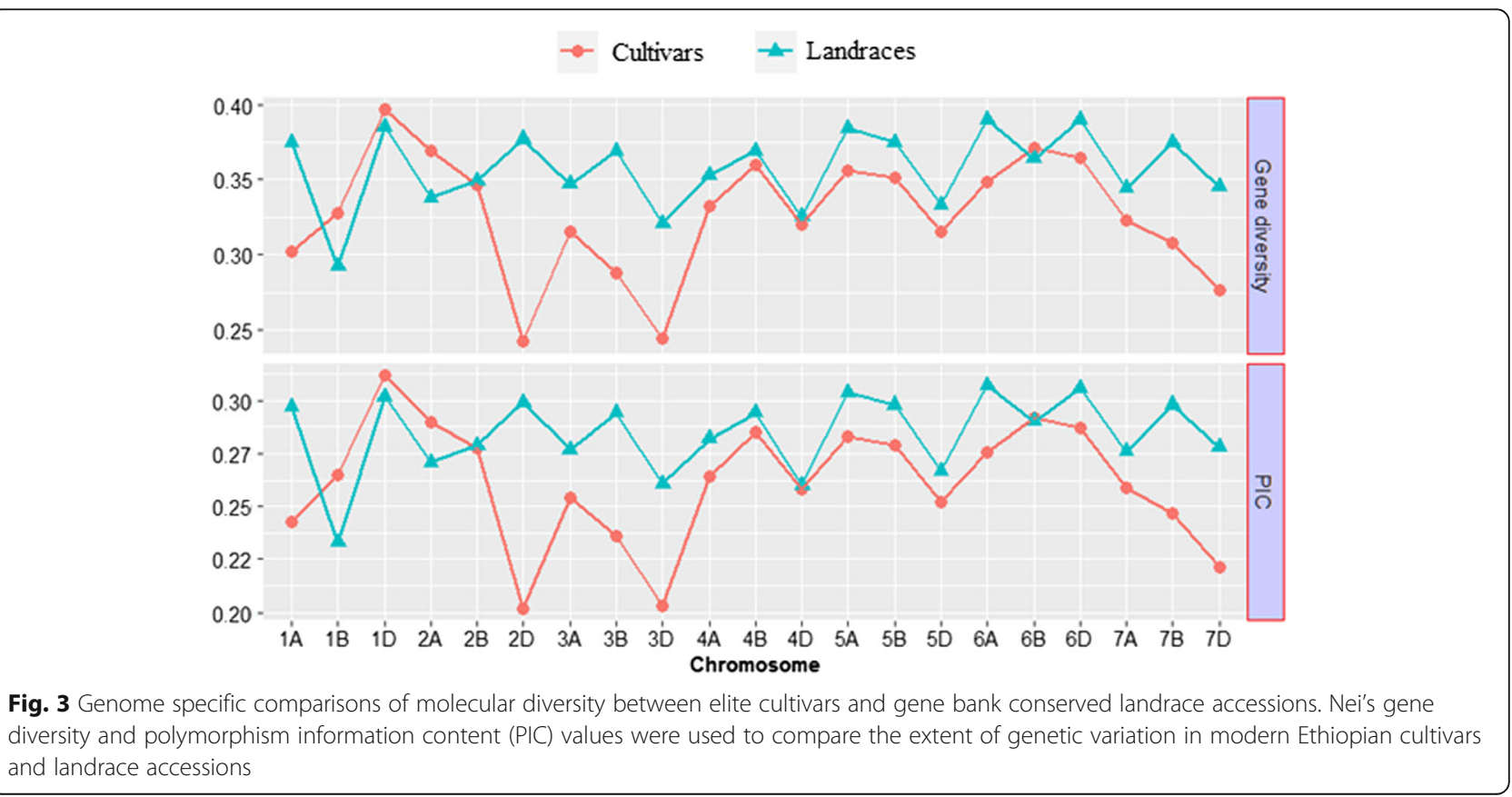




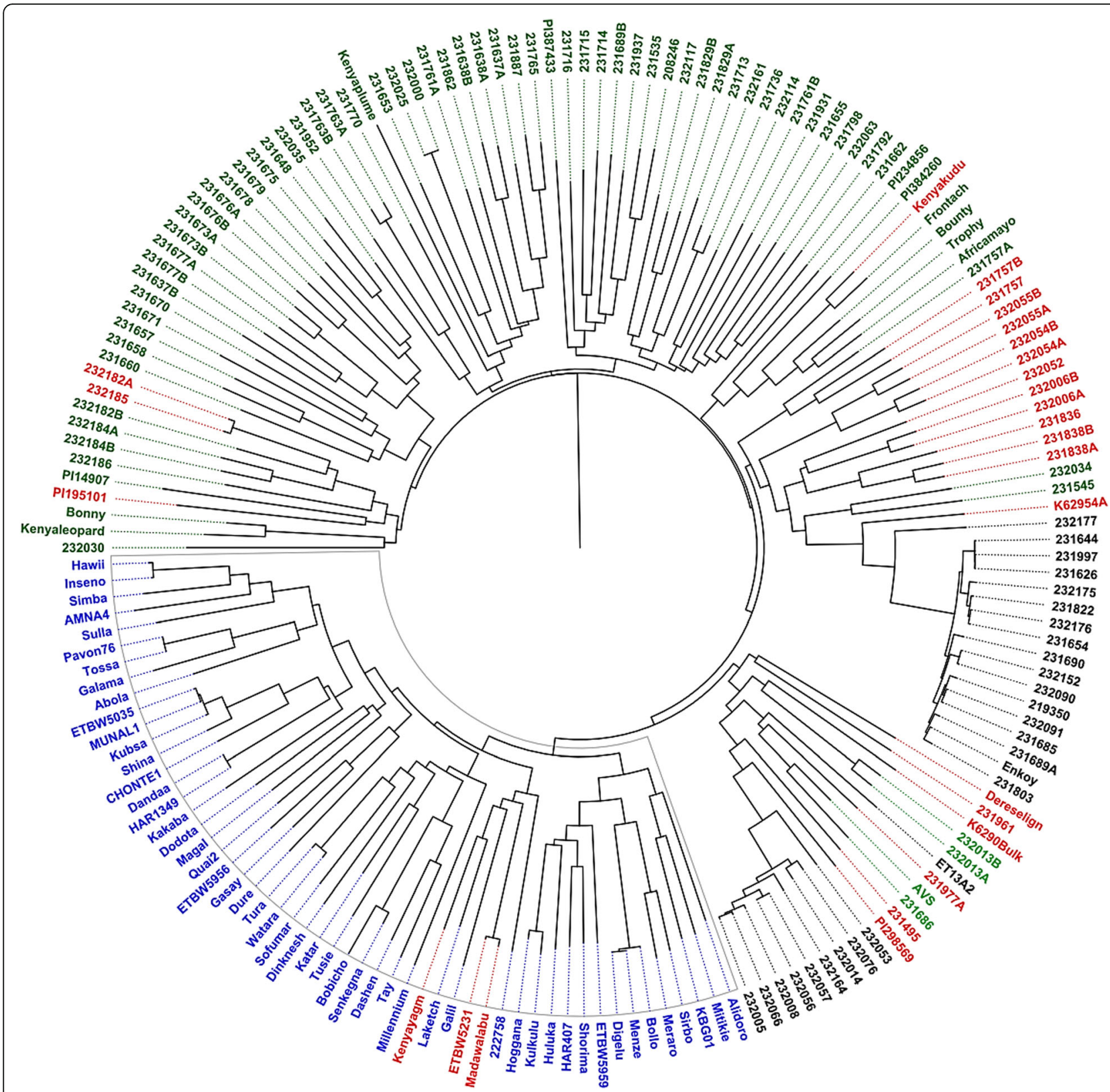

Fig. 4 Dendrogram of the Ethiopian wheat accessions estimated by shared-allele genetic distance using high-density SNP markers. Cluster analysis was based on the neighbor-joining algorithm. Accessions have been assigned colors based on STRUCTURE analysis at $\mathrm{K}=3$. Black $=$ sub-population 1 (SP_I), Green = sub-population 2 (SP_II (i)) and Blue = subpopulation 3 (SP_II (ii)). Structure analysis indicated the likely number of sub-population to be two (SP_I and SP_II). Separate analysis of population structure within SP_I showed no sign of further sub-division, while SP_II subdivided into SP_II (i) and SP_II (ii). The blue colored accessions (SP_II (ii) in the structure analysis) are Ethiopian cultivars, while the rest are landraces

$\left(r^{2}>0.18\right)$. In the A genome, the percentage of marker pairs that were in LD greater than 0.18 for chromosome $1 \mathrm{~A}$ was the highest $(6.8 \%)$, while in the $\mathrm{B}$ genome chromosome $1 \mathrm{~B}$ contained the highest percentage of marker pairs (11.0\%) that showed LD greater than the critical $r^{2}$ value. For the $\mathrm{D}$ genome, the percentage of SNP pairs that showed LD greater than 0.18 for chromosome $2 \mathrm{D}$ was the highest (4.2\%).
LD decayed to the critical $r^{2}$ value (0.18) at about $2.5 \mathrm{cM}$, which was used to determine the confidence interval for declaring distinct MTAs (Fig. 6).

\section{Association analysis for field-based and seedling resistance to Pst}

Genome-wide association analyses were performed for stripe rust IT and DS within each of the eight 


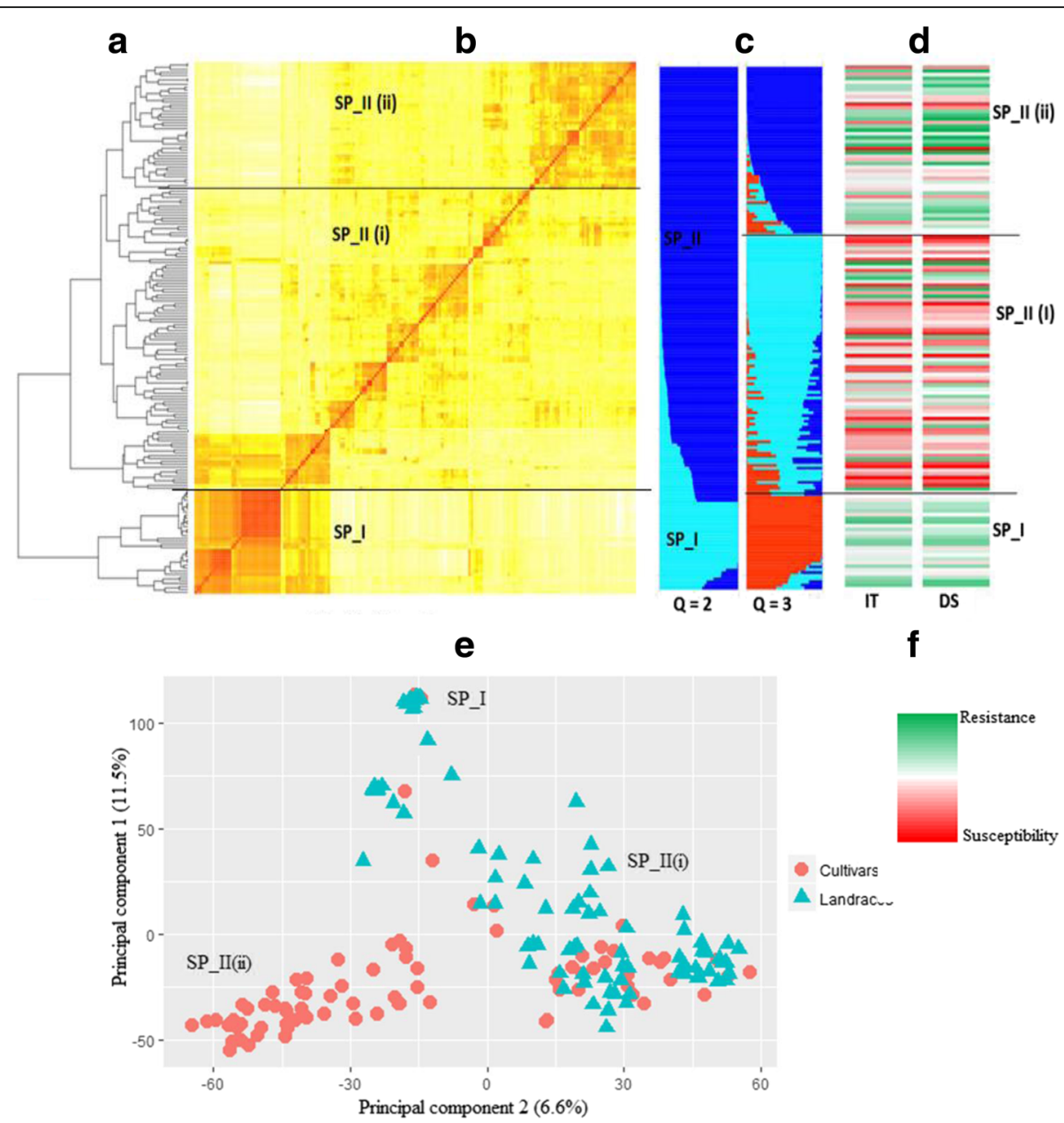

Fig. 5 Genetic relatedness, population structure and the relationship between population sub-clustering and stripe rust resistance. a The distance based hierarchical clustering of the accessions based on the Fast Ward grouping algorithm. b Heat map of identity-by-decent (IBD) kinship matrix. c Clustering diagrams of population structure based on the model based quantitative assessment of subpopulation membership. $\mathbf{d}$ Heat map of reactions of the accessions to stripe rust IT and DS based on BLUP values across all environments. e Principal component analysis (PCA). f Color key for the heat map of the phenotypes. SP_I and SP_II are sub-populations defined according to the optimum number of clusters determined by STRUCTURE analysis. SP-II was further sub-grouped into two (SP-II (i) and SP-II (ii)) based on kinship analysis that agrees with the STRUCTURE analysis when $\mathrm{K}=3$ was considered. Accessions in SP-II (ii) are all recent and historical Ethiopian bread wheat cultivars

environments. Based on the assessment of the performance of the GWAS models for controlling population structure and kinship, the K + PC3 CMLM model (containing the first three principal components and a compressed kinship) gave a minimum deviance of observed $P$ values from expected distribution in Q-Q plot (Additional file 4) and was used for all GWAS analyses. The analyses revealed 67 genomic regions significantly associated with adult plant IT or DS at nominal probability $(P)<0.005$ in at least four test environments. A confidence interval for linkage blocks of the 67 regions was determined based on average LD decay rate $( \pm 2.5 \mathrm{cM})$ and adjacent markers were assigned to the linkage blocks based on the significance and consistency of associations across environments. Markers with the most significant associations to each trait were used as the MTA-tagging marker. Altogether, the 67 genomic regions explained 54.3\% and $52.1 \%$ of the total variation in IT and DS, respectively (excluding variation explained by the population structure). Additional file 5 summarizes detailed information of the 67 putative resistance loci.

Eleven of the 67 genomic regions were significant at False Discovery Rate (FDR) [52] adjusted $P<0.1$ (Table 2). The 11 high-probability QTL were located on chromosomes 1B, 2A, 4A, 5A, 6A and 7B. The phenotypic variation $\left(R^{2}\right)$ explained by these genomic regions were in the range of $3-11 \%$. When combined, these loci explained 27.5 and $26.9 \%$ of the total variation in Pst IT and DS responses, respectively. Results of the GWAS tests are discussed in detail focusing on 


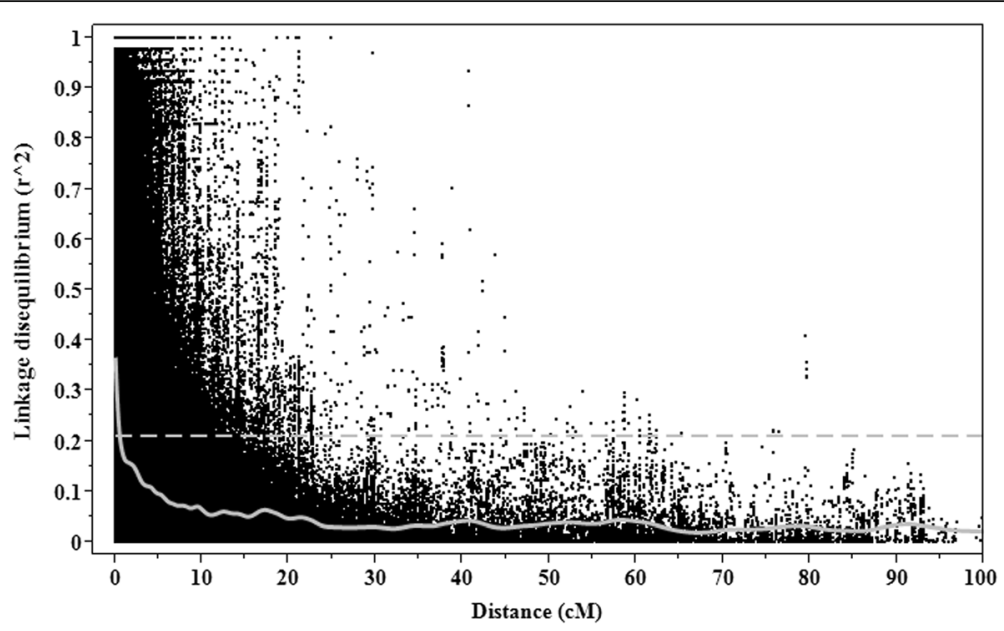

Fig. 6 Genome-wide linkage disequilibrium decay plot for the Ethiopian wheat accessions based on high-density SNP markers. LD, measured as $r^{2}$ between pairs of polymorphic marker loci is plotted against the genetic distance (cM) between the markers. LOESS smoothening curve (solid line) and mean LD (broken line) were fitted to the LD decay

the chromosome regions with significance at FDRadjusted $P<0.1$.

Among the 11 highly significant genomic regions, a block of haplotypes covering a genetic distance from 65 to $74 \mathrm{cM}$ on chromosome $1 \mathrm{~B}$ was identified to harbor major Pst resistance QTL/genes. SNPs IWB11553 (mapped at $65 \mathrm{cM}$ ) and IWB9661 (mapped at $74 \mathrm{cM}$ ) represented loci significantly associated with stripe rust IT and DS on 1BS (Table 2). The phenotypic variation explained by $I W B 11553$ and IWB9661 loci were in the range of $3.1-9.6 \%$ and $3.4-6.7 \%$, respectively.

On the short arm of chromosome $2 \mathrm{~A}$, a cluster of significant SNPs covering a genetic distance of 9.4-47.0 cM was detected. Among these, IWB11136 (mapped at $9.4 \mathrm{cM}$ ), IWB6584 (mapped at $26 \mathrm{cM}$ ) and IWB57199 (mapped at $47.0 \mathrm{cM}$ ) showed strong associations (FDR $P<0.1)$ across multiple locations and growing seasons. The phenotypic variation explained by IWB11136, IWB6584 and IWB57199 loci ranged from 3.5-8.3\%, 3.1$11.2 \%$ and $3.4-9.5 \%$, respectively. Each SNP was in strong LD with each other $\left(r^{2}=0.60,0.68\right.$ and 0.88 between IWB11136 and IWB6584, IWB11136 and IWB57199; and IWB6584 and IWB57199, respectively), in spite of extended genetic distance in the consensus map.

On chromosome 4A, SNP IWB24187 (located $114.5 \mathrm{cM}$ ) was among the 11 strongly associated markers representing effective stripe rust resistance loci. The amount of variation explained by IWB24187 ranged 2.86.1\%. On chromosomes 5A, SNP markers IWA1280 and IWB28837, mapped at $15.7 \mathrm{cM}$ and $123 \mathrm{cM}$, were significant with $R^{2}$ values in the range of 2.7-6.1 and 3.0-7.1\%, respectively. Several other SNP markers that were mapped close to IW1280 and IWB28837 were also significant at nominal probability of association $(P<0.005$ across multiple locations and growing seasons), but unable to pass the stringent criteria based on FDR-adjusted $P<0.1$.

On the long arm of chromosome 6A, SNP IWB69846 (mapped at $136.9 \mathrm{cM}$ ) also showed strong association with Pst IT and DS. The amount of variation explained by the resistance loci associated with IWB69846 ranged from $3.1-8.3 \%$. Two resistance loci were identified on chromosome 7B, associated with SNP markers IWA3506 (mapped on the short arm at $57 \mathrm{cM}$ ) and IWB58601 (mapped on the long arm at $155.4 \mathrm{cM}$ ). The resistance locus tagged by IWA3506 was detected as significant both in the seedling and adult plant tests with an average $R^{2}$ value of $6 \%$ at adult stage and $11.4 \%$ at seedling stage. The resistance locus tagged by IWB58601 was effective across multiple locations and seasons at adult plant stage and showed average $R^{2}$ value of $4.8 \%$.

Seedling resistance to Pst detected by GWAS among the Ethiopian wheat accessions is summarized in Table 2. Associations significant at FDR-adjusted $P$ value $<0.1$ were detected for eight genomic regions on chromosomes $1 \mathrm{~B}$, $2 \mathrm{~A}, 2 \mathrm{~B}, 2 \mathrm{D}, 5 \mathrm{~B}$ and $7 \mathrm{~B}$. The phenotypic variation explained by each of the eight regions ranged from 9.4 to 13.8\%. Among these, IWB 9661 (mapped to chromosome $1 \mathrm{~B}$ at $74.4 \mathrm{cM}$ ), IWB6584 and IWB57199 (mapped to chromosome $2 \mathrm{~A}$ at $26 \mathrm{cM}$ ) and IWA3506 (mapped to chromosome $7 \mathrm{~B}$ at $56.9 \mathrm{cM}$ ) were commonly detected in the seedling and adult plant tests. On chromosome 2B, SNP IWB43797 (located at $134.5 \mathrm{cM}$ ) was significantly associated with seedling resistance to PSTv- 41 and PSTv106. On chromosome 2D, SNP IWB10441 showed strong association with resistance to PSTv-106. Two FDR $P<0.1$ significant genomic regions were detected on chromosome $5 \mathrm{~B}$ for seedling resistance to Pst, which were represented by SNPs IWB14332 (mapped at $29.1 \mathrm{cM}$ ) and 


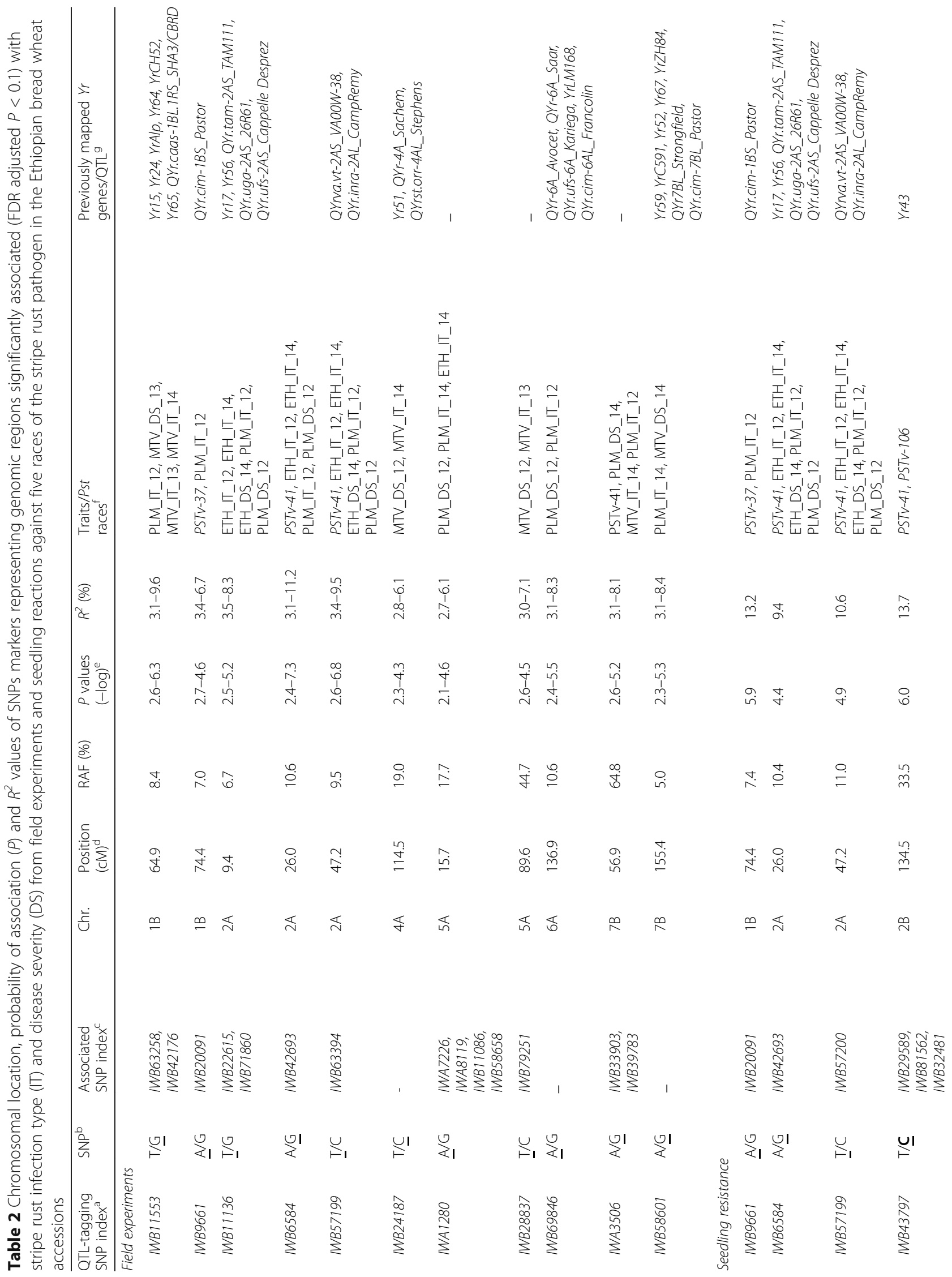




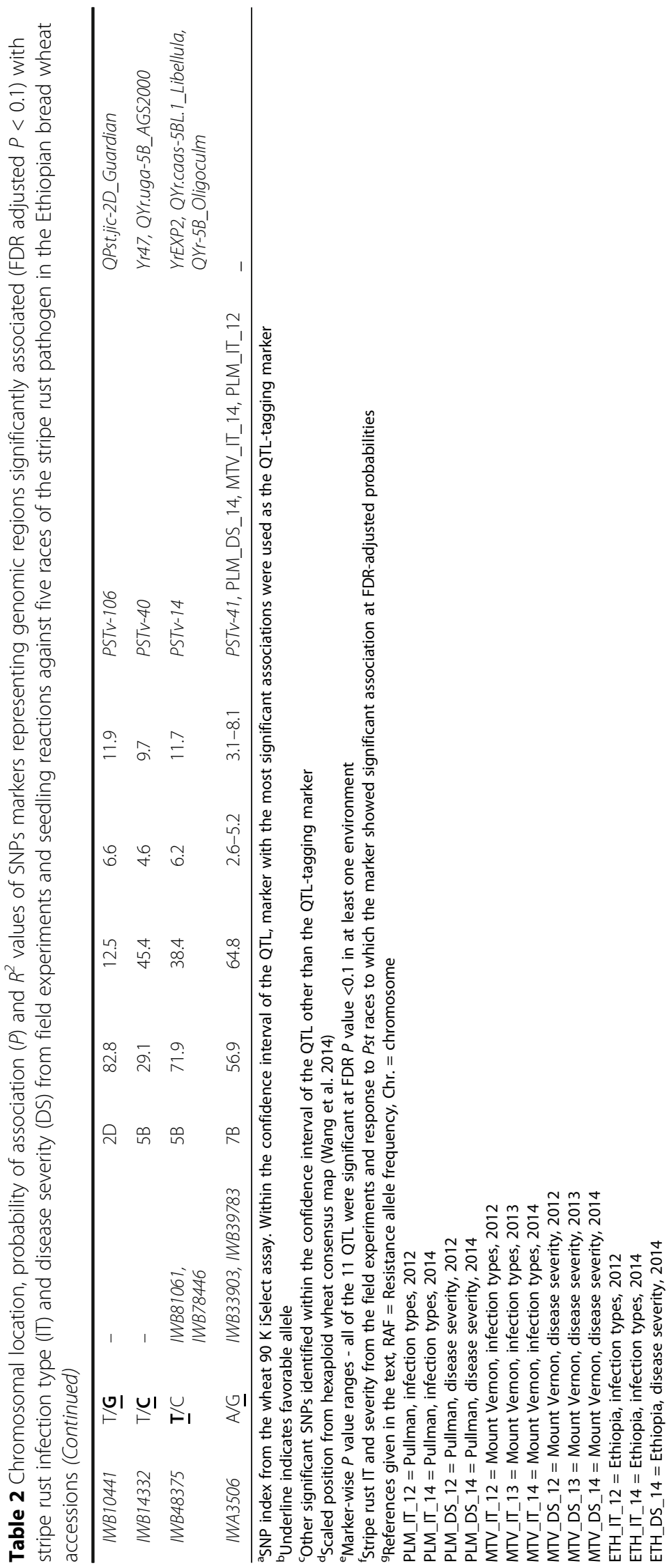


IWB48375 (mapped at $72 \mathrm{cM}$ ). The LD between the two genomic regions was non-significant $\left(r^{2}<0.001\right)$, indicating that they represent two different resistance loci.

\section{Mapping of seedling resistance to Pgt}

GWAS identified nine genomic regions significantly associated with seedling response to the four tested races of Pgt (Table 3). Among these, a resistance locus mapped to the short arm of chromosome 2B was significantly associated with response to race TTKSK. This locus was represented by SNP IWB26389, which was mapped at $99.9 \mathrm{cM}$ on 2BS. IWB26389 explained $8.0 \%$ of the phenotypic variance in the germplasm panel. One additional genomic region on 7B, IWB664169 at $171.1 \mathrm{cM}$, was significantly associated with race TTKSK. This genomic region accounted for about $2.4 \%$ of the total variation in IT response against TTKSK. GWAS of seedling resistance to race TTTTF identified a single genomic region (represented by IWB34733) that was mapped to the long arm of chromosome $4 \mathrm{~A}$ at $144.4 \mathrm{cM}$. The phenotypic variance explained by $I W B 34733$ was $15 \%$. The locus tagged by IWB34733 was also effective against race TKTTF. Five genomic regions were significantly associated with seedling resistance to race TRTTF. These putative resistance loci were mapped to chromosomes $1 \mathrm{~B}, 2 \mathrm{~A}, 3 \mathrm{~B}, 6 \mathrm{~A}$ and $7 \mathrm{~B}$. The five genomic regions were tagged by IWB39306 (chromosome $1 \mathrm{~B}$ at $60.6 \mathrm{cM}$ ), IWB12320 (chromosome 2A at $106.3 \mathrm{cM}$ ), IWB19479 (chromosome 3B at $136.3 \mathrm{cM}$ ), IWA5416 (chromosome 6A at $5.6 \mathrm{cM}$ ) and IWB2378 (chromosome $7 \mathrm{~B}$ at $92.5 \mathrm{cM}$ ). The phenotypic variance explained by the genomic regions significant for race TRTTF ranged $5.1 \%$ to $6.4 \%$. On chromosome $6 \mathrm{~B}$, a genomic region tagged by SNP marker IWB14375 showed highly significant association with response to race TKTTF. This genomic region was responsible for 9\% of the phenotypic variation. IWB14375 was significantly associated also with resistance to races TTTTF and TRTTF.

\section{Validation of previously mapped rust resistance genes}

Genotyping of the accessions with molecular markers linked to previously mapped stripe rust and stem rust resistance genes/QTL revealed the presence of $L r 34 /$ Yr18/Sr57, Yr17/Sr38, Sr31/Yr9/Pm8/Lr26, Sr2/Yr30, $\operatorname{Sr} 36$ and $\operatorname{Sr} 24$ at a frequency of $12.0 \%, 6.2 \%, 9.0 \%, 13 \%$, $30 \%$ and $2.6 \%$, respectively (Additional files 3 and 6 ). A single accession (Alidoro) was positive for the resistance-associated alleles of both $L r 34 / Y r 18 / \operatorname{Sr} 57$ and $Y r 17 / S r 38$, while four lines (Sofumer, Tusie, Bobicho and AMNA-4) carry both $L r 34 / Y r 18 / S r 57$ and Sr31/Yr9/Lr26. Three accessions (Sofumer, Bonny, and $231,658)$ carried both $L r 34 / Y r 18 / S r 57$ and $\mathrm{Yr} 30 / \mathrm{Sr} 2$, while only one accession (KBG-01) was positive for the resistance-associated alleles of both $\mathrm{Yr} 17 / \mathrm{Sr} 38$ and $\mathrm{Yr} 30 / \mathrm{Sr} 2$. Four accessions identified as landraces $(231,658,232,034$ and 232,014) carried the resistance alleles of both $\mathrm{Yr} 30 / \mathrm{Sr} 2$ and $\mathrm{Sr} 36$. Accession 231,658 carried $L r 34 / Y r 18 / S r 57, Y r 30 / S r 2$ and Sr36. None of the accessions were positive for the $L r 67 / Y r 46$ gene. Association analysis revealed a significant association of the $L r 34 / Y r 18$ KASP marker wMAS000003 with field resistance to stripe rust at marker wise $P$ value $<0.005$ in at least half of the test environments. However, no significant SNPs were detected on this region of chromosome

Table 3 Genomic regions significantly associated with seedling responses to the four races of Puccinia graminis $\mathrm{f}$. sp. tritici in the Ethiopian bread wheat accessions

\begin{tabular}{|c|c|c|c|c|c|c|c|c|}
\hline QTL-tagging SNP index & SNP & Associated SNPs index & Chr. & Position (cM) & MAF & $P$ value & $R^{2}(\%)$ & FDR $P$ values \\
\hline \multicolumn{9}{|l|}{$\overline{T R T T F}$} \\
\hline IWB39306 & $\underline{\mathbf{A}} / \mathrm{G}$ & - & $1 \mathrm{~B}$ & 60.6 & 0.1 & 1.30E-04 & 5.08 & * \\
\hline IWB12320 & $\underline{T} / \mathrm{C}$ & - & $2 \mathrm{~A}$ & 106.3 & 0.15 & 3.40E-05 & 5.98 & * \\
\hline IWB19479 & $\underline{T} / \mathrm{G}$ & IWB1595 & $3 B$ & 136.3 & 0.07 & $7.20 \mathrm{E}-05$ & 5.47 & * \\
\hline IWA5416 & $\underline{T} / \mathrm{C}$ & IWB11315, IWB22036, IWB60233, IWB67415 & $6 \mathrm{~A}$ & 5.6 & 0.08 & $2.00 \mathrm{E}-05$ & 6.35 & * \\
\hline IWB2378 & $\mathrm{T} / \underline{\mathbf{C}}$ & IWB4726, IWB4725, IWB7743, IWB65791 & $7 \mathrm{~B}$ & 92.5 & 0.19 & $2.00 \mathrm{E}-05$ & 6.35 & * \\
\hline \multicolumn{9}{|l|}{ TTKSK } \\
\hline IWB26389 & $\underline{\mathbf{A}} / \mathrm{G}$ & IWB42742, IWB1518, IWB24614, IWB32327 & $2 B$ & 99.9 & 0.31 & $2.40 \mathrm{E}-10$ & 8.02 & $* * * *$ \\
\hline IWB64169 & $\underline{\mathbf{A}} / \mathrm{G}$ & IWB63221 & $7 \mathrm{~B}$ & 171.1 & 0.13 & $3.00 \mathrm{E}-04$ & 2.41 & $* *$ \\
\hline \multicolumn{9}{|l|}{ TTाTF } \\
\hline IWB34733 & $\mathrm{A} / \underline{\mathbf{G}}$ & IWB63979, IWB61381, IWB59018 & $4 \mathrm{~A}$ & 144.4 & 0.08 & $8.90 \mathrm{E}-10$ & 14.62 & $* * * *$ \\
\hline \multicolumn{9}{|l|}{ TKTTF } \\
\hline IWB14375 & $\mathbf{T} / \underline{G}$ & IWB59006, IWB72471, IWB59005 & $6 \mathrm{~B}$ & 113.7 & 0.096 & 3.60E-07 & 0.09 & $* *$ \\
\hline
\end{tabular}

$$
\begin{aligned}
& * * * *=<0.0001 \\
& { }^{* * *}<0.001 \\
& { }^{* *}<0.05 \\
& { }^{*}<0.1
\end{aligned}
$$


7D and none of the SNPs on the 7D chromosome were in significant LD with the KASP marker. Significant associations were also detected for $\mathrm{Yr} 17 / \mathrm{Lr} 37 / \mathrm{Sr} 38 \mathrm{KASP}$ marker together with several other SNP markers including IWB11136, IWB6584, IWB42693, IWB30196, IWB57199 and $I W B 63394$ that were in LD with the $\operatorname{Yr} 17$ marker Ventriup-LN2.

Although there was no significant MTA detected for $Y r 9$ KASP marker 1RS.1BL_6110 in association with Pst resistance, the associated $\mathrm{Sr}$ gene (Sr31) showed an effect of reducing IT responses to races TRTTF, TTTTF and TKTTF by $44 \%, 50 \%$ and $45 \%$, respectively. As expected, accessions postulated to carry the resistance allele of $\operatorname{Sr} 31$ were highly susceptible to TTKSK. The resistance-associated allele of the marker for Sr24 was carried by cultivars Huluka, Shorima, Hoggana, Millennium, and Kulkullu in this panel, and showed highly resistant responses to all races of the stem rust pathogen. Accessions carrying Sr36 showed highly effective responses to TTKSK, but were mostly susceptible to TKTTK and TTTTF.

\section{Discussion \\ Phenotypic variability and molecular diversity of the Ethiopian bread wheat accessions}

A better understanding of the extent of genetic diversity and genetic basis of the responses to rust diseases in wheat may offer the prospect of effective exploitation of genetic resources and enhanced breeding for durable rust resistance in wheat. To this end, the current study surveyed Ethiopian bread wheat landraces and cultivars for resistance to Pst and Pgt under diverse environments and variable pathogen populations. Our data revealed that the accessions, most notably the landraces, possess considerable variation for resistance to both diseases. The sources of resistance identified with high levels of combined resistance to stripe rust and stem rust may be used as parental breeding lines for improving wheat resistance to these diseases. Profiling of the accessions with high-density SNP markers facilitated analyses of genome-specific molecular diversity and phylogeny between the landraces and elite cultivars as well as a genome-wide survey of marker-trait associations. It was evident that the landrace accessions were characterized by high levels of molecular diversity as measured by Nei's gene diversity index, PIC values, NJ clustering algorithms and kinship analyses. By contrast, the Ethiopian bread wheat elite cultivars show high genetic similarity, with some of them nearly genetically redundant. Some of these nearly genetically redundant cultivars were released by different breeding programs and are widely grown across Ethiopia, which demonstrates one of the likely reasons for the frequent failures of deployed resistance genes and highlights the continued risk of disease epidemics due to minimal diversity. The loss of genetic diversity in modern wheat varieties compared to landraces has been reported by previous studies $[19,20,53]$. Such erosion of genetic variation in the elite wheat varieties may constrain the capacity to counter threats from changing pathogen populations. A particular concern in this regard is the fact that the highlands of Ethiopia are 'hot spot' areas for the Triticum-Puccinia pathosystem $[16,54,55]$ and therefore the presence of high inoculum pressure and rapid changes in pathogen virulence. Cultivation of genetically uniform wheat cultivars and the practice of frequent double cropping in some wheat growing areas of the country may result in rapid selection for virulent races of the pathogen. The results of our analyses of genetic diversity in Ethiopian bread wheat cultivars highlight the continued risk of wheat rust epidemics due to the genetic vulnerability of the current wheat varieties and suggest the utilization of more genetic diversity to broaden the genetic base of elite wheat cultivars. We also believe that Ethiopian landraces are a unique source of unexploited diversity for wheat improvement [27].

\section{MTAs for Pst resistance and co-localization of the putative QTL with previously identified $\mathrm{Yr}$ genes/QTL}

The mixed model analyses of association between the high-density genome-wide SNP markers and response to Pst in the Ethiopian bread wheat accessions highlighted several chromosome regions harboring putative resistance loci. Eleven FDR $P<0.1$-significant regions, perhaps of large effect, were identified when employing the most stringent criteria. Four additional genomic regions were significantly associated (FDR-adjusted $P<0.10$ ) with seedling resistance to stripe rust. Aiming to keep a reasonable power of statistical association and identify loci conferring intermediate effects, less stringent GWAS tests based on marker-wise significance tests were also considered. The latter approach was supported by associations detected across multiple location and seasons. Fifty-six additional Pst resistance loci, at a marker-wise $P<0.005$ in at least half of the test environments, were detected. The amount of variation explained by the 67 genomic regions ranged from 54.3 to $52.1 \%$. The reason for the partial account of the variation in the field response to stripe rust by the significant markers could be attributed to the fact that the current GWAS methods are more suited to the identification of common variants of large to intermediate effects [56]. In addition, the $90 \mathrm{~K}$ iSelect chip did not provide enough marker coverage in the $\mathrm{D}$ genome to identify all Pst resistance loci present in the germplasm panel. It was evident that none of the $90 \mathrm{~K} \mathrm{SNP} \mathrm{markers}$ mapped to the short arm of chromosome 7D were linked to the marker for known Pst resistance gene $\mathrm{Yr} 18$ ( Lr34/Yr18/Pm38) included as a control in a separate 
GWAS that showed experiment-wise significant associations with Pst resistance at multiple locations.

The percent of variation explained by the 56 MTAs from the field experiment support that many are unlikely false-positives. Nonetheless, the details of only the 11 highest-confidence resistance-associated MTAs from the field experiment and four MTAs from the greenhouse seedling resistance screening are further discussed.

To determine whether any known resistance genes coincided with the 15 putative resistance regions identified in this study, the current results were compared with the integrated genetic maps of Pst resistance genes and SNP markers constructed by Maccaferri et al. [24] and Bulli et al. [26]. The genomic regions harboring eight of the eleven loci identified from our field studies of stripe rust resistance overlap with positions of previously reported QTL. Among the three potentially novel resistance loci, IWB28837 and IWA1280 were associated with resistance on chromosomes $5 \mathrm{~A}$ at 15.7 and $89.6 \mathrm{cM}$ positions, respectively. In line with their distant map positions, IWB28837 and IWA1280 are not in LD with each other, and thus tag distinct genomic regions. The confidence intervals of MTAs tagged by these two SNPs do not overlap with positions of any of the previously reported QTL or Yr genes. Thus, IWA1280 and IWB28837 likely represent novel resistance loci for Pst. On the short arm of 7B, IWA3506 (at $57.0 \mathrm{cM}$ ) was significant in both seedling and adult plant tests. Yr63 has been mapped to the short arm of 7B chromosome (McIntosh et al., 2013). Based on the relative genetic position of Yr63 in the integrated genetic map constructed by [24], IWA3506 was mapped at an average genetic distance of $30.0 \mathrm{cM}$ from $\mathrm{Yr} 63$, which indicates that IWA3506 is likely different from $\mathrm{Yr} 63$, and thus represents a newly documented stripe rust resistance locus.

The haplotype blocks of significant stripe rust resistance loci detected on chromosome 1B spanning a segment of 63-74 cM likely harbor more than one resistance locus. Figure $7 \mathrm{a}$ illustrates LD relationships between the two loci on 1BS (IWB11553 at $64.9 \mathrm{cM}$ and IWB9661 at $74.4 \mathrm{cM}$ ), which were all less than $r^{2}=0.03$. This indicates that $I W B 11553$ and IWB9661 likely represent distinct genomic regions. Chromosome 1B is rich with $Y r$ genes/QTL, and a number of $Y r$ genes have been mapped to the proximity of the genomic regions tagged by IWB9314, IWB11553 and IWB 9661 , including Yr15, Yr24/Yr26/YrCh42, YrAlp, YrCH52, Yr52, Yr64 and Yr65. Other Pst resistance QTL were also previously mapped in this region of chromosome 1B, including QYr.caas-1BL.1RS_SHA3/CBRD and QYr.cim-1BS_Pastor [57-61]. Additional research is needed to fully characterize chromosome $1 \mathrm{~B}$ resistance.

We detected significant associations between stripe rust resistance and SNP markers mapped close to the translocation segment harboring $\mathrm{Yr} 17$ including the
Yr17 marker Ventriup-LN2. Since virulence to $\mathrm{Yr} 17$ is widespread [29, 30], the significant association of $\mathrm{Yr} 17$ and other SNP markers may indicate the presence of another effective resistance gene tightly linked to $\mathrm{Yr} 17$. Among the three significant SNPs detected, IWB11136 (located at 9.4 $\mathrm{cM}$ ) was in perfect $\mathrm{LD}\left(r^{2}=1.0\right)$ with the Yr17 KASP marker. IWB6584 and IWB57199 were mapped at the 26 and $47 \mathrm{cM}$ positions, respectively and in LD with the $\operatorname{Yr} 17$ KASP marker with $r^{2}$ value of 0.64 and 0.68 , respectively (Fig. 7b). Hence, IWB6584 and IWB57199 may not be located on the 2NS.2AS translocation segment carrying $\operatorname{Yr} 17$, but tightly linked and likely represent a gene responsible for the high level of resistance exhibited by lines positive for the favorable alleles of the SNPs. Several other temporarily designated QTL have been identified on the short arm of chromosome 2A, including QYr.sun-2A_Wollaroi [62], QYr.tam2AS_TAM111 [63], QYrva.vt-2AS_VA00W-38 [64], QYr.inra_2A1_Recital [65] and QYr.ucw-2A_PI610750 [66]. IWB6584 and IWB57199 could be related to these QTL. Tan et al. (2013) reported mapping of a QTL on 2AS encoding 12-oxo-phytodienoic acid reductase (OPR) that is collinear to an $\mathrm{Yr} 17$-containing-fragment of the chromosome arm. The genes encoding OPR are known to play critical roles in insect and disease resistance pathways in higher plants $[67,68]$. Further genetic analysis is required to determine the relationship of MTAs detected by IWB6584 and IWB57199 with previously mapped genes/QTL.

On chromosome 6A, an MTA associated with field resistance to Pst was identified at FDR-adjusted probability $(P)<0.1$ at multiple locations and seasons. This locus was tagged by the SNP IWB69846 at the map position of $136.9 \mathrm{cM}$. Previously, YrLM168 [69] and other temporarily designated QTL [QYr-6A_Avocet [70], QYr-6A_Saar [71], QYr.ufs-6A_Kariega [72] and QYr.cim-6AL_Francolin [73] have been mapped to the vicinity of $I W B 69846$, and could be related to the locus tagged by IWB69846. On chromosome 4A, IWB24187 represents an MTA mapped closely to $\operatorname{Yr} 51$ [74] and two other QTL, QYr-4A_Sachem [75] and QYrst.orr4AL_Stephens [76]. Further genetic analysis will be required to determine the relationship between the resistance locus tagged by IWB24187 and the previously mapped QTL/genes.

On the long arm of chromosome 7B, SNP marker IWB58601 was associated with Pst resistance across multiple field tests. The confidence interval of the genomic region tagged by IWB58601 overlaps with the map positions of $\operatorname{Yr} 59, \operatorname{YrC591}, \operatorname{Yr} 52, \operatorname{Yr} 67, \mathrm{YrZH84}$ and QTL, QYr.cim-7BL_Pastor, QYr7BL_Strongfield [58, 77, 78]. Yr52 and Yr59 confer high-temperature adult-plant (HTAP) resistance to stripe rust, while $Y r C 591, Y r 67$ and YrZH84 are dominant genes conferring seedling resistance 


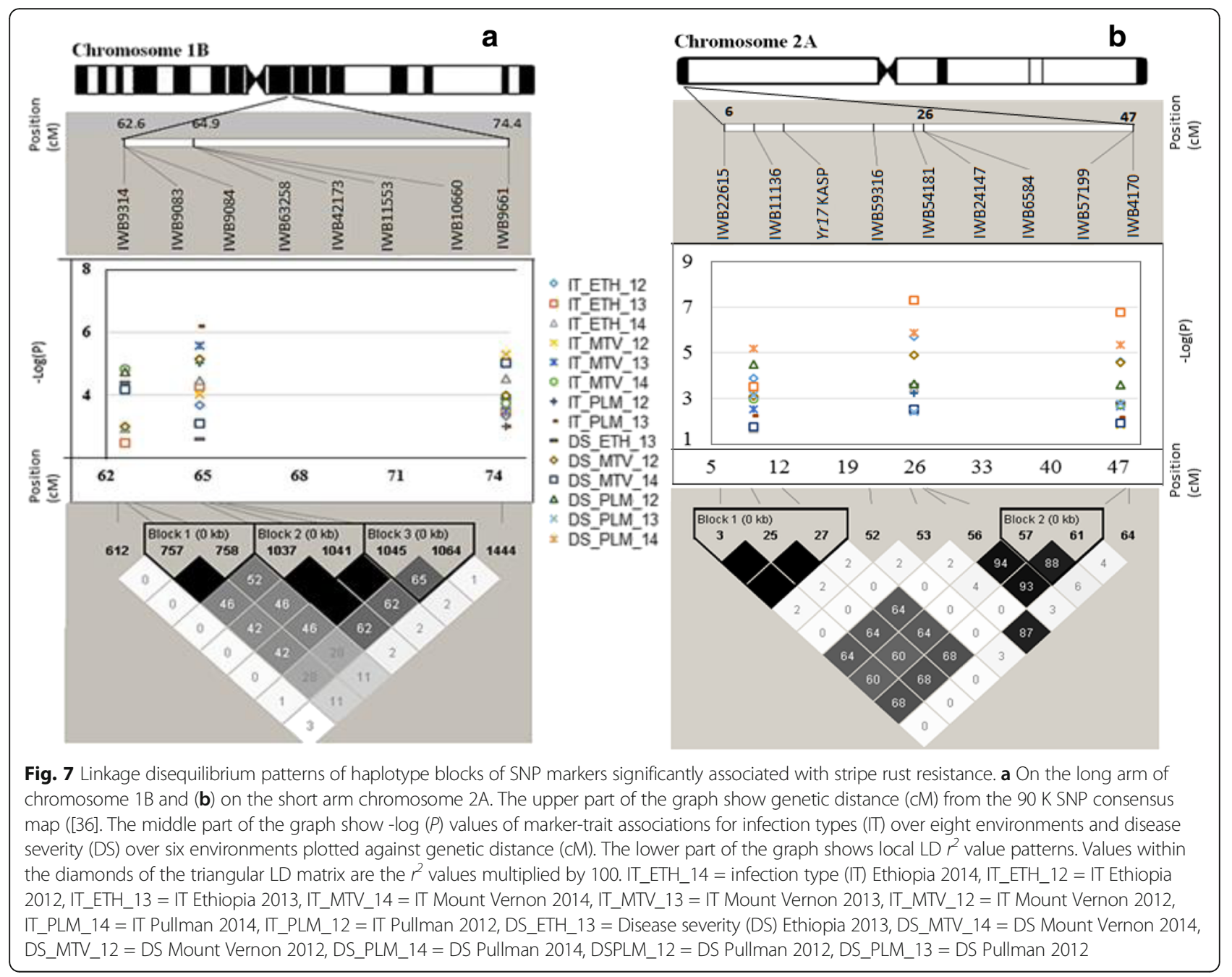

to stripe rust. It has been reported that IWB37096 and IWB71995, both mapped at $148.7 \mathrm{cM}$ on 7BL [36], are flanking $Y r 67$ with a respective genetic distance of 1.1 and $0.6 \mathrm{cM}$ [79]. In the present study, these two SNPs (IWB37096 and IWB71995) were not among the 67 significant MTAs and in non-significant LD with IWB58601, suggesting that the QTL tagged by IWB58601 may be different from those of IWB37096 and IWB71995 as well as Yr63. However, since these SNPs and the previously mapped resistance genes are located close to each other, further characterization and genetic analysis is required to determine whether they are distinct from those previously mapped resistance genes.

\section{MTAs for Pgt resistance and co-localization of the} resistance loci with previously identified $\mathrm{Sr}$ genes/QTL

Five genomic regions were significantly associated with seedling resistance to race TKTTF. Among these, IWB39306 was mapped close to the segment of 1BS that harbors the $1 \mathrm{BL} .1 \mathrm{RS}$ rye translocation carrying
Sr31 [80]. Sr31 is ineffective to TTKSK, but confers effective resistance to TRTTF and TKTTF $[16,33]$. To determine if $I W B 39306$ is related to $\mathrm{Sr} 31$, we genotyped the accessions with a KASP marker for 1BL.1RS and performed LD analysis. IWB39306 and the KASP marker for 1BL.1RS were in complete LD, indicating that IWB39306 is detecting allelic variation based on the presence or absence of 1BL.1RS. The accessions carrying the resistance-associated allele of IWB39306 are all elite Ethiopian cultivars. Working on the North American spring wheat breeding germplasm, Bajgain et al. [81] identified 13 significant SNPs on the short arm of chromosome 1B (position range 44-65 cM) that also showed various levels of significant associations with resistance to race TRTTF in our study, but did not pass the FDR threshold of $<0.1$. We found strong LD between the SNP markers identified by Bajgain et al. [81], Sr31 and IWB39306 ( $r^{2}$ ranging from 0.41 to 1.00$)$. This further clarifies the uncertainty whether the 13 significant markers are linked to $S r 31$, or to a novel gene of resistance to TRTTF. 
The SNP locus IWB12320 on chromosome 2A at $106.3 \mathrm{cM}$ was significantly associated with response to race TRTTF. Eighteen of the 25 accessions carrying the resistance-associated allele of $I W B 12320$ are landraces. Stem rust resistance gene $\mathrm{Sr} 21$ was mapped approximately $50 \mathrm{cM}$ from the centromere on the long arm of 2A [82, 83]. Sr21 confers resistance to several races of Pgt, including those in the Ug99 group. However, Sr21 is derived from $T$. monococcum and was later transferred to hexaploid wheat [82]. Hence, Sr21 is not common in landrace accessions, and suggests that IWB12320 is tagging a resistance locus or allele distinct from $\mathrm{Sr} 21$. Stem rust resistance genes $\mathrm{Sr} 48$ and $\mathrm{SrTm} 4$ have also been previously mapped to the long arm of chromosome $2 \mathrm{~A}$ $[84,85]$. However, IWB12320 may not be related to $\operatorname{SrTm} 4$, as the latter was identified from the diploid wheat, Triticum monococcum, and is not common in hexaploid wheat. $\mathrm{Sr} 48$ (first designated as $\mathrm{SrAnl}$ ) is a seedling resistance gene that was mapped to chromosome arm 2AL of the bread wheat cultivar Arina. Based on the genetic map position of $S r 48$ ( $\sim 55 \mathrm{cM}$ from the centromere), it is likely that the genomic region tagged by $I W B 12320$ corresponds to Sr48. Allelism tests will be required to test the relationships between IWB12320 and $\mathrm{Sr} 48$.

On 3BL, SNP IWB19479 (mapped at $136.3 \mathrm{cM}$ ) identified a genomic region significantly associated with the response to race TRTTF. This locus may represent a new resistance gene since no race TRTTF-effective seedling resistance genes have been mapped to chromosome 3B so far. Similarly, no TTKSK-effective seedling resistance genes have been identified on chromosome 7B [86]. Therefore, the significant genomic region detected on chromosome $7 \mathrm{~B}$ in the present study (IWB2378 mapped at $92.5 \mathrm{cM}$ ) is likely tagging a new resistance gene effective to TTKSK. On the short arm of chromosome 6A, SNP IWA5416 (mapped at $5.6 \mathrm{cM})$ tagged a genomic region significantly associated with resistance to TRTTF. This 6AS locus is most likely linked to the gene $\operatorname{Sr} 8 a$, which is effective to race TRTTF [87, 31]. Bajgain et al. [81] identified a significant association of IWA5416 and 56 other SNP markers with race TRTTF, validating the association of this genomic region with resistance to Pgt.

Analysis of response to TTTTF identified a significant genomic region that was mapped to long arm of chromosome 4A. This locus was identified by SNP IWB34733, mapped at $144.4 \mathrm{cM}$, and was also effective to TTKSK and TRTTF, but did not pass the FDR threshold of significant associations for these races. It is likely that IWB34733 is related to $\mathrm{Sr7a}$. Bajgain et al. [81] also identified 51 significant SNPs mapped on 4AL, spanning 142-164 cM, in North American spring wheat breeding germplasm, one of which was IWB34733.
A single genomic region was detected that was significantly associated with race TKTTF, a newly detected Ethiopian Pgt race that defeated resistance in the popular cultivar 'Digalu' [16]. SNP marker IWB14375, mapped at $113.7 \mathrm{cM}$ on $6 \mathrm{~B}$, identified this genomic region. This region of chromosome 6B is known to harbor Sr11, which is effective against TKTTF [86]. Bajgain et al. [81] also detected significant association of IWB14375 with response to race TKTTF in North American spring wheat germplasm. Nirmala et al. [88] identified seven SNP markers linked to Sr11. Among the seven SNP markers, IWB59006 and IWB72471 also showed highly significant association with the response to TKTTF in the present study. Both $I W B 59006$ and IWB72471 were in strong LD $\left(r^{2}=0.8-\right.$ 1.0) with $I W B 14375$, indicating that the locus tagged by IWB14375 corresponds to Sr11.

A genomic region on the short arm of chromosome 2B, tagged by IWB26389, was significantly associated with IT response to race TTKSK. Although the short arms of 2A, 2B and 2D are known to carry Sr32 [79], it is unlikely that the three markers represent $\mathrm{Sr} 32$ as it is not common in landrace accessions due to its origin from Ae. speltoides and relatively recent translocations to hexaploid wheat. The stem rust resistance gene Sr36 derived from Triticum timopheevi is also located on 2BS and confers resistance against TTKSK [89]. To determine if IWB26389 is associated with Sr36, we determined LD between the KASP marker for Sr36 (wMAS000015) and IWB26389. The result showed that IWB26389 and Sr36 KASP marker are in complete LD, which indicates that IWB26389 is also tagging the T. timopheevi segment that harbors $\operatorname{Sr} 36$.

We identified a genomic region on 7BL, tagged by IWB64169 at $171 \mathrm{cM}$, significantly associated with response to race TTKSK. Although there are several QTL effective to Ug99 on chromosome 7B, no effective seedling resistance gene has been identified so far [86]. Therefore, the significant genomic region detected on $7 \mathrm{BL}$ in the present study may be a new resistance gene effective to TTKSK. Based on LD analysis, IWB64169 represents a different genomic region from IWB2378, which was significant to race TRTTF.

\section{Conclusion}

The limited pool of alleles present in the Ethiopian bread wheat cultivars may offer narrow perspective for germplasm improvement and consequently limit the capacity to deal with threats from wheat rust epidemics. Notwithstanding, enough genetic variation resides within the landrace accessions that can be utilized to broaden the genetic base of rust resistance in wheat breeding germplasm. In addition to characterizing the genetic diversity of the Ethiopian bread wheat cultivars and landraces, this study highlights the potential of GWAS to accelerate 
the translation of landrace germplasm diversity toward applied wheat improvement. Several newly documented resistance loci were discovered from Ethiopian landraces, along with detection of previously reported resistance genes. The molecular markers linked to resistance loci identified in the current study can be used to efficiently select for resistance to diversify the genetic basis of elite cultivars.

\section{Additional files}

Additional file 1: Virulence/avirulence formula of Puccinia striiformis $\mathrm{f}$. sp. tritici isolates used for seedling resistance screening of the Ethiopian wheat accessions. (XLSX $10 \mathrm{~kb}$ )

Additional file 2: Pearson's correlation coefficients among stripe rust infection type (IT) and disease severity (DS) in each location and season. (XLSX $12 \mathrm{~kb}$ )

Additional file 3: Infection types and disease severity observed on Ethiopian wheat cultivars and landraces tested with races of Puccinia striiformis f. sp. tritici and Puccinia graminis f. sp. tritici at seedling stage and evaluated under field conditions. Allele report for markers linked to previously known stripe rust and stem rust resistance genes and SNP markers significantly associated with the resistance to both diseases (identified in this study) are summarized. (XLSX $50 \mathrm{~kb}$ )

Additional file 4: Comparison of different association test models using Quantile-Quantile (Q-Q) plots using BLUP values of stripe rut IT and DS across all environments. The Q-Q plot determines the magnitude of the deviation of the observed association between the markers and response to stripe rust from the expected null hypothesis of no association. Under the assumption of no association between the SNPS and the traits, a large inflation of observed $P$ values from the expected $P$ values indicates spurious associations. Only few true SNP-trait associations are expected to deviate from the null hypothesis. (DOCX $95 \mathrm{~kb}$ )

Additional file 5: Genomic regions significantly associated with field resistance of the Ethiopian bread wheat accessions to Puccinia striiformis f. sp. tritici at marker wise $P<0.005$ in at least four of the eight environments. (XLSX $15 \mathrm{~kb}$ )

Additional file 6: Frequency of favorable alleles of the molecular markers linked to previously mapped stripe rust and stem rust resistance genes/QTL in the Ethiopian wheat accessions. (DOCX 16 kb)

\section{Abbreviations}

APR: Adult plant resistance; CMLM: Compressed mixed linear model; DS: Disease severity; GLM: General linear model; GWAS: Genome-wide association study; IT: Infection types; LD: Linkage disequilibrium; MAF: Minor allele frequency; MLM: Mixed linear model; MTA: Marker-trait association; PC: Principal component; PCA: Principal component analysis; Pgt: Puccinia graminis f. sp. tritici; PNW: Pacific Northwest; Pst: Puccinia striiformis f. sp. tritici; Q-Q: quantile-quantile; QTL: Quantitative trait locus; SNP: Single nucleotide polymorphism

\section{Acknowledgments}

We would thank our colleagues at the Ethiopian Institute of Agricultural Research (EIAR), Kulumsa Agricultural Research Center (KARC) and the International Maize and Wheat Improvement Center (CIMMYT), Ethiopia Office for providing the necessary resources and technical support during the field experiments in Ethiopia. We also thank John Kuehner, Victor DeMacon and Dr. Kent Evans for providing technical support in the field and greenhouse.

\section{Competing of interests}

The authors declare that they have no competing interests.

\section{Funding}

This project was partly funded by Monsanto Beachell-Borlaug International Scholars Program, by the National Research Initiative Competitive Grant 2011-68,002-30,029 (Triticeae-CAP) from the United States Department of Agriculture National Institute of Food and Agriculture, and by the Durable Rust Resistance in Wheat Project with funds through Cornell University originating from the Bill and Melinda Gates Foundation and the Department for International Development of the United Kingdom.

\section{Availability of data and materials}

The data sets generated or analyzed during this study are included in this published article and its additional files. The phylogenetic tree and genotypic data used to perform the neighbor-joining phylogeny analysis are deposited in TreeBASE and are available at: http://purl.org/phylo/treebase/phylows/ study/TB2:S21219

\section{Authors' contributions}

KTM MP and XC conceived and designed the experiments. KTM MP MR SR XC BGB performed the experiments. KTM carried out the statistical analysis, interpretation of the results, and drafted the manuscript. MP MR XC BGB revised the paper. All authors read and approved the final manuscript.

Ethics approval and consent to participate

Not applicable.

\section{Consent for publication}

Not applicable.

\section{Publisher's Note}

Springer Nature remains neutral with regard to jurisdictional claims in published maps and institutional affiliations.

\section{Author details}

${ }^{1}$ Department of Crop and Soil Sciences, Washington State University, Pullman, WA 99164-6420, USA. USDA-ARS Cereal Disease Laboratory, Department of Plant Pathology, University of Minnesota, St. Paul, MN 55108, USA. ${ }^{3}$ USDA-ARS, Wheat Health, Genetics, and Quality Research Unit, and Department of Plant Pathology, Washington State University, Pullman, WA 99164-6430, Pullman, WA 99164-6430, USA. ${ }^{4}$ Ethiopian Institute of Agricultural Research, Kulumsa Agricultural Research Center, P. O. Box 489, Assela, Ethiopia.

Received: 15 December 2016 Accepted: 21 July 2017

Published online: 04 August 2017

\section{References}

1. Chen XM. Epidemiology and control of stripe rust [Puccinia striiformis $\mathrm{f}$. Sp. tritici ] on wheat. Can. J. Bot. 2005;27:314-37.

2. Kolmer JA. Tracking wheat rust on a continental scale. Curr Opin Plant Biol. 2005;8:441-9.

3. Singh RP, Huerta-espino J, William HM. Genetics and breeding for durable resistance to leaf and stripe rusts in wheat. Turk J Agric. 2005;29:121-7.

4. Hovmøller MS, Yahyaoui AH, Milus EA, Justesen AF. Rapid global spread of two aggressive strains of a wheat rust fungus. Mol Ecol. 2008:17:3818-26.

5. Milus EA, Kristensen K, Hovmøller MS. Evidence for increased aggressiveness in a recent widespread strain of Puccinia striiformis $\mathrm{f} s p$ tritici causing stripe rust of wheat. Phytopathology. 2009;99:89-94.

6. Solh M, Nazari K, Tadesse W, Wellings CR. The growing threat of stripe rust worldwide. Borlaug Glob. Rust Initiat. Tech. Work. 1-4 Sept. 2012, Beijing, China. 2012;1-10.

7. Global Rust Reference Center. http://wheatrust.org/. Accessed 15 July 2016.

8. Mcintosh RA, Brown GN. Anticipatory breeding for resistance to rust diseases in wheat. Annu Rev Phytopathol. 1997:35:311-26.

9. Singh RP, Hodson DP, Jin Y, Lagudah ES, Ayliffe MA, Bhavani S, et al. Emergence and spread of new races of wheat stem rust fungus : continued threat to food security and prospects of genetic control. Phytopathology. 2015:105:872-84.

10. Pretorius ZA, Singh RP, Wagoire WW, Payne TS. Detection of virulence to wheat stem rust resistance gene Sr31 in Puccinia graminis. f. Sp. tritici in Uganda. Plant Dis. 2000;84:203. 
11. Jin Y, Szabo LJ, States U, Agricultural A, Disease C. Detection of virulence to resistance gene Sr24 within race TTKS of Puccinia graminis f. Sp. tritici. Plant Dis. 2008:92:923-6.

12. Jin Y, Szabo LJ, States U, Agricultural A, Disease C, Paul S, et al. Detection of virulence to resistance gene Sr36 within the TTKS race lineage of Puccinia graminis f. Sp. tritici. Plant Dis. 2009:93:367-70.

13. Rouse MN, Nirmala J, Pretorius ZA, Hiebert CW. Characterization of Srgh, a wheat stem rust resistance allele effective to Ug99. Theor Appl Genet. 2014; 127:1681-8.

14. Patpour M, Hovmøller MS, Justesen AF, Newcomb M, Olivera P, Jin Y, et al. Emergence of virulence to SrTmp in the Ug99 race Group of Wheat Stem Rust, Puccinia graminis f. Sp. tritici, in Africa. Plant Dis. 2016;100:522.

15. Patpour M, Hovmoller MS, Shahin AA, Newcomb M, Olivera P, Jin Y, et al. First report of the Ug99 race Group of Wheat Stem Rust, Puccinia graminis $f$ Sp. tritici, in Egypt in 2014. Plant Dis. 2016;100:863.

16. Olivera P, Newcomb M, Szabo LJ, Rouse M, Johnson J, Gale S, et al. Phenotypic and Genotypic Characterization of Race TKTTF of Puccinia graminis f. sp. tritici that caused a wheat stem rust epidemic in southern Ethiopia in 2013-14. Phytopathology. 2015;105:917-28.

17. Burdon JJ, Barrett LG, Rebetzke G, Thrall PH. Guiding deployment of resistance in cereals using evolutionary principles. Evol Appl. 2014;7:609-24.

18. Hulbert S, Pumphrey M. A time for more booms and fewer busts? Unraveling cereal-rust interactions. Mol Plant-Microbe Interact. 2014;27:207-14.

19. Haudry A, Cenci A, Ravel C, Bataillon T, Brunel D, Poncet C, et al. Grinding up wheat : a massive loss of nucleotide diversity since domestication. Mol Biol Evol. 2007;24:1506-17

20. Peng JH, Sun D, Nevo E. Domestication evolution, genetics and genomics in wheat. Mol Breed. 2011;28:281-301.

21. Feuillet $C$, Langridge $P$, Waugh $R$. Cereal breeding takes a walk on the wild side. Trends Genet. 2007;24:24-32

22. Vasudevan K, Vera Cruz CM, Gruissem W, Bhullar NK. Large scale germplasm screening for identification of novel rice blast resistance sources. Front Plant Sci. 2014;5:1-9.

23. Zurn JD, Newcomb M, Rouse MN, Jin Y, Chao S, Sthapit J, et al. High-density mapping of a resistance gene to Ug99 from the Iranian landrace PI 626573. Mol Breed. 2014:1-11.

24. Maccaferri M, Zhang J, Bulli P, Abate Z, Chao S, Cantu D, et al. A Genome-Wide Association Study of Resistance to Stripe Rust (Puccinia striiformis f. sp. tritici) in a Worldwide Collection of Hexaploid Spring Wheat (Triticum aestivum L.). G3; Genes|Genomes|Genetics. 2015:5:449-65.

25. Kertho A, Mamidi S, Bonman JM, McClean PE, Acevedo M. Genome-wide association mapping for resistance to leaf and stripe rust in winter-habit hexaploid wheat landraces. PLoS One. 2015;10:e0129580.

26. Bulli P, Zhang J, Chao S, Chen X, Pumphrey M, Health W. Genetic architecture of resistance to stripe rust in a global winter wheat germplasm collection. G3 Genes|Genomes|Genetics. 2016;6:2237-53.

27. Mengistu DK, Kidane YG, Catellani M, Frascaroli E, Fadda C, P ME. High-density molecular characterization and association mapping in Ethiopian durum wheat landraces reveals high diversity and potential for wheat breeding. Plant Biotechnol. 2016; doi:10.1111/pbi.12538.

28. Line RF, Qayoum A. Virulence, aggressiveness, evolution, and distribution of races of Puccinia striiformis (the cause of stripe rust of wheat) in North America. United States dep. Agric Tech Bull. 1968-1987;1992:44.

29. Wan A, Chen X. Virulence characterization of Puccinia striiformis f. Sp. tritici using a new set of Yr single-gene line differentials in the United States in 2010. Plant Dis. 2014;98:1534-42.

30. Wan A, Muleta KT, Zegeye H, Hundie B, Pumphrey M, Chen X. Virulence characterization of wheat stripe rust fungus Puccinia striiformis $\mathrm{f}$. Sp. tritici in ethiopia and evaluation of ethiopian wheat germplasm for resistance to races of the pathogen from Ethiopia and the United States. Plant Dis. 2017;101:73-80.

31. Olivera PD, Jin Y, Rouse M, Badebo A, Fetch T, Singh RP, et al. Races of Puccinia graminis f. Sp. tritici with combined virulence to Sr13 and Srge in a field stem rust screening nursery in Ethiopia. Plant Dis. 2012;96:623-8.

32. Jin Y, Sigh RP, Ward RW, Wanyera R, Kinyua M, Njau P, et al. Characterization of seedling infection types and adult plant infection responses of monogenic Sr gene lines to race TTKS of Puccinia graminis f. Sp. tritici. Plant Dis. 2007;91:1096-9.

33. Rouse MN, Jin Y. Stem rust resistance in A-genome diploid relatives of wheat. Plant Dis. 2011;95:941-4.

34. Stakman EC, Steward DM, Loegering WQ. Identification of physiologic races of Puccinia graminis var. tritici. US Dep. Agric. Agric. Res. Serv. 1962:E-617.
35. Zhang D, Bowden R, Bai G. A method to linearize Stakman infection type ratings for statistical analysis. Proc. 2011 Borlaug Glob. Rust Initiat. Tech. Work. 17-20 June 2011, St. Paul, MN. 2011;P. 28.

36. Wang S, Wong D, Forrest K, Allen A, Chao S, Huang BE, et al. Characterization of polyploid wheat genomic diversity using a high-density 90000 single nucleotide polymorphism array. Plant Biotechnol. 2014;12:787-96.

37. Marker Assisted Selection in Wheat (MAS Wheat). http://maswheat.ucdavis. edu/Index.htm. Accessed 20 May 2016. 2016;2016.

38. Forrest K, Pujol V, Bulli P, Pumphrey M, Wellings C, Herrera-Foessel S, et al. Development of a SNP marker assay for the Lr67 gene of wheat using a genotyping by sequencing approach. Mol Breed. 2014;34:2109-18.

39. Rasheed A, Wen W, Gao F, Zhai S, Jin H, Liu J, et al. Development and validation of KASP assays for genes underpinning key economic traits in bread wheat. Theor. Appl. Genet. Springer. Berlin Heidelberg. 2016;129:1843-60.

40. Mago R, Bariana HS, Dundas IS, Spielmeyer W, Lawrence GJ, Pryor AJ, et al. Development of PCR markers for the selection of wheat stem rust resistance genes Sr24 and Sr26 in diverse wheat germplasm. Theor Appl Genet. 2005;111:496-504.

41. Liu K, Muse SV. PowerMarker : an integrated analysis environment for genetic marker analysis. Oxford Univ. Presstime. 2005;21:2128-9.

42. Weir BS. Genetic data analysis II methods for discrete population genetic data. Sundarland, MA: Sinauer Assoc; 1996.

43. Felsenstein J. PHYLIP ( Phylogeny Inference Package ) Version 3.66. Dep. Genome Sci. Univ. Washingt. Seattle, WA. 2006.

44. Pritchard JK, Stephens M, Donnelly P. Inference of population structure using multilocus genotype data. Genet Soc Am. 2000;155:945-59.

45. Evanno G, Regnaut S, Goudet J. Detecting the number of clusters of individuals using the software structure: a simulation study. Mol Ecol. 2005; 14:2611-20.

46. Cleveland WS. Robust locally weighted regression and smoothing scatterplots. J Am Stat Assoc. 1979;74:829-36.

47. Breseghello, Sorrells FM. Association Mapping of Kernel Size and Milling Quality in Wheat (Triticum aestivum L.) Cultivars. Genetics. 2005;172:1165-1177.

48. Corbeil RR, Searle SR. Restricted maximum likelihood ( REML) estimation of variance components in the mixed model. Technometrics. 1976;18:31-8.

49. Yu J, Pressoir G, Briggs WH, Vroh Bi I, Yamasaki M, Doebley JF, et al. A unified mixed-model method for association mapping that accounts for multiple levels of relatedness. Nat Genet. 2006;38:203-8.

50. Zhang Z, Ersoz E, Lai CQ, Todhunter RJ, Tiwari HK, Gore MA, et al. Mixed linear model approach adapted for genome-wide association studies. Nat Genet. 2010;42:355-60.

51. Lipka AE, Tian F, Wang Q, Peiffer J, Li M, Bradbury PJ, et al. GAPIT: genome association and prediction integrated tool. Bioinformatics. 2012;28:2397-9.

52. Benjamini $Y$, Hochberg $Y$. Controlling the false discovery rate: a practical and powerful approach to multiple testing. J R Stat Soc. 1995:289-300.

53. Ren J, Chen L, Sun D, You FM, Wang J, Peng Y, et al. SNP-revealed genetic diversity in wild emmer wheat correlates with ecological factors. BMC Evol Biol. 2013:13:1-15

54. Negassa M. Estimates of phenotypic diversity and breeding potential of Ethiopian wheats. Hereditus. 1986:8:41-8.

55. Tadesse K, Ayalew A, Badebo A. Effect of fungicide on the development of wheat stem rust and yield of wheat varieties in highlands of Ethiopia. African Crop Sci J. 2010;18:23-33.

56. Korte A, Farlow A. The advantages and limitations of trait analysis with GWAS: a review. Plant Methods. 2013:9:29.

57. Ma J, Zhou R, Dong Y, Wang L, Wang X, Jia J. Molecular mapping and detection of the yellow rust resistance gene $Y_{r 2} 6$ in wheat transferred from Triticum turgidum L. using microsatellite markers. Euphytica. 2001; 120:219-26.

58. Li ZF, Zheng TC, He ZH, Li GQ, Xu SC, Li XP, et al. Molecular tagging of stripe rust resistance gene YrZH84 in Chinese wheat line Zhou 8425B. TAG Theor Appl Genet. 2006;112:1098-103.

59. Ren RS, Wang MN, Chen XM, Zhang ZJ. Characterization and molecular mapping of Yr52 for high-temperature adult-plant resistance to stripe rust in spring wheat germplasm PI 183527. Theor Appl Genet. 2012;125: 847-57.

60. Rosewarne GM, Singh RP, Huerta-Espino J, Herrera-Foessel SA, Forrest KL, Hayden MJ, et al. Analysis of leaf and stripe rust severities reveals pathotype changes and multiple minor QTLs associated with resistance in an avocet $\times$ pastor wheat population. Theor Appl Genet. 2012;124:1283-94. 
61. Cheng P, Xu LS, Wang MN, See DR, Chen XM. Molecular mapping of genes Yr64 and Yr65 for stripe rust resistance in hexaploid derivatives of durum wheat accessions PI 331260 and PI 480016. Theor Appl Genet. 2014;127: 2267-77.

62. Bansal UK, Kazi AG, Singh B, Hare RA, Bariana HS. Mapping of durable stripe rust resistance in a durum wheat cultivar Wollaroi. Mol Breed. 2014:33:51-9.

63. Basnet BR, Ibrahim AMH, Chen X, Singh RP, Mason ER, Bowden RL, et al. Molecular mapping of stripe rust resistance in hard red winter wheat TAM 111 adapted to the U.S. High Plains. Crop Sci. 2014;54:1361-73.

64. Christopher MD, Liu S, Hall MD, Marshal DS, Fountain MO, Johnson JW, et al. Identification and mapping of adult-plant stripe rust resistance in soft red winter wheat cultivar USG 3555. Plant Breed. 2013;132:53-60.

65. Dedryver F, Paillard S, Mallard S, Robert O, Trottet M, Nègre S, et al. Characterization of genetic components involved in durable resistance to stripe rust in the bread wheat "Renan". Phytopathology. 2009;99:968-73.

66. Lowe I, Cantu D, Dubcovsky J. Durable resistance to the wheat rusts: integrating systems biology and traditional phenotype-based research methods to guide the deployment of resistance genes. Euphytica. 2011;179:69-79.

67. Zhang J, Simmons C, Yalpani N, Crane V, Wilkinson H, Kolomiets M. Genomic analysis of the 12-oxo-phytodienoic acid reductase gene family of Zea mays. Plant Mol Biol. 2005;59:323-43.

68. Tan CT, Carver BF, Chen M, Gu Y, Yan L. Genetic association of OPR genes with resistance to hessian fly in hexaploid wheat. BMC Genomics. 2013;14:1-11.

69. Feng J, Chen G, Wei Y, Liu Y, Jiang Q, Li W, et al. Identification and genetic mapping of a recessive gene for resistance to stripe rust in wheat line LM168-1. Mol Breed. 2014;33:601-9.

70. William HM, Singh RP, Huerta-Espino J, Palacios G, Suenaga K. Characterization of genetic loci conferring adult plant resistance to leaf rust and stripe rust in spring wheat. Genome. 2006;49:977-90.

71. Lillemo M, Asalf B, Singh RP, Huerta-Espino J, Chen XM, He ZH, et al. The adult plant rust resistance loci $L r 34 / Y r 18$ and $L$ r46/Yr29 are important determinants of partial resistance to powdery mildew in bread wheat line Saar. Theor Appl Genet. 2008;116:1155-66.

72. Prins $R$, Pretorius ZA, Bender CM, Lehmensiek A. QTL mapping of stripe, leaf and stem rust resistance genes in a Kariega $x$ avocet $S$ doubled haploid wheat population. Mol Breed. 2011:27:259-70.

73. Suenaga K, Singh RP, Huerta-Espino J, William HM. Microsatellite markers for genes $L$ r34/Yr18 and other quantitative trait loci for leaf rust and stripe rust resistance in bread wheat. Phytopathology. 2003;93:881-90.

74. Randhawa M, Bansal U, Valarik M, Klocova B, Dolezel J, Bariana H. Molecular mapping of stripe rust resistance gene $\mathrm{Yr} 51$ in chromosome $4 \mathrm{AL}$ of wheat Theor Appl Genet. 2014;127:317-24

75. Singh A, Pandey MP, Singh AK, Knox RE, Ammar K, Clarke JM, et al. Identification and mapping of leaf, stem and stripe rust resistance quantitative trait loci and their interactions in durum wheat. Mol Breed. 2013:31:405-18

76. Vazquez MD, Peterson CJ, Riera-Lizarazu O, Chen X, Heesacker A, Ammar K, et al. Genetic analysis of adult plant, quantitative resistance to stripe rust in wheat cultivar "Stephens" in multi-environment trials. Theor Appl Genet. 2012;124:1-11

77. Xu H, Zhang J, Zhang P, Qie Y, Niu Y, Li H, et al. Development and validation of molecular markers closely linked to the wheat stripe rust resistance gene YrC591 for marker-assisted selection. Euphytica. 2014; 198:317-23

78. Zhou XL, Wang MN, Chen XM, Lu Y, Kang ZS, Jing JX. Identification of Yr59 conferring high-temperature adult-plant resistance to stripe rust in wheat germplasm PI 178759. Theor Appl Genet. 2014;127:935-45.

79. Mcintosh R, Yamazaki Y, Dubcovsky J, Rogers WJ, Morris C, Appels R, et al. Catalogue of gene symbols for wheat in $12^{\text {th }}$ international wheat genetics symposium. Mclntosh, Yokohama, Japan: Ed. by R.A; 2013

80. Mago R, Spielmeyer W, Lawrence GJ, Lagudah ES, Ellis JG, Pryor A. Identification and mapping of molecular markers linked to rust resistance genes located on chromosome 1RS of rye using wheat-rye translocation lines. Theor Appl Genet. 2002;104:1317-24.

81. Bajgain P, Rouse MN, Bulli P, Bhavani S, Gordon T, Wanyera R, et al. Association mapping of north American spring wheat breeding germplasm reveals loci conferring resistance to Ug99 and other African stem rust races. BMC Plant Biol. 2015:15:1-19.
82. The $\Pi$. Chromosome location of genes conditioning stem rust resistance transferred from diploid to hexaploid wheat. Nat New Biol. 1973:241:256.

83. Chen S, Rouse MN, Zhang W, Jin Y, Akhunov E, Wei Y, et al. Fine mapping and characterization of Sr21, a temperature-sensitive diploid wheat resistance gene effective against the Puccinia graminis f. Sp. tritici Ug99 race group. Theor. Appl. Genet. 2015;128:645-56.

84. Bansal UK, Bossolini E, Miah H, Keller B, Park RF, Bariana HS. Genetic mapping of seedling and adult plant stem rust resistance in two European winter wheat cultivars. Euphytica. 2008;164:821-8.

85. Briggs J, Chen S, Zhang W, Nelson S, Dubcovsky J, Rouse MN. Mapping of SrTm4, a recessive stem rust resistance gene from diploid wheat effective to Ug99. Phytopathology. 2015;105:1347-54.

86. Yu LX, Barbier H, Rouse MN, Singh S, Singh RP, Bhavani S, et al. A consensus map for Ug99 stem rust resistance loci in wheat. Theor Appl Genet. 2014; 127:1561-81.

87. Letta T, Olivera P, Maccaferri M, Jin Y, Ammar K, Badebo A, et al. Association mapping reveals novel stem rust resistance loci in durum wheat at the seedling stage. Plant Genome. 2014;7:1-13.

88. Nirmala J, Chao S, Olivera P, Babiker EM, Abeyo B, Tadesse Z, et al. Markers Linked to Wheat Stem Rust Resistance Gene Sr11 Effective to Puccinia graminis f. sp. tritici Race TKTTF. Phytopathology. 2016;106:1352-8.

89. Rouse MN, Nava IC, Chao S, Anderson JA, Jin Y. Identification of markers linked to the race Ug99 effective stem rust resistance gene Sr28 in wheat (Triticum aestivum L.). Theor. Appl. Genet. 2012;125:877-85.

\section{Submit your next manuscript to BioMed Central and we will help you at every step:}

- We accept pre-submission inquiries

- Our selector tool helps you to find the most relevant journal

- We provide round the clock customer support

- Convenient online submission

- Thorough peer review

- Inclusion in PubMed and all major indexing services

- Maximum visibility for your research

Submit your manuscript at www.biomedcentral.com/submit
) Biomed Central 